\title{
Nucleon self-energy in the relativistic Brueckner approach
}

\author{
L. Sehn, C. Fuchs, Amand Faessler \\ Institut für Theoretische Physik, Auf der Morgenstelle 14, 72076 Tübingen
}

\begin{abstract}
The formalism of the relativistic (or Dirac-) Brueckner approach in infinite nuclear matter is described. As nucleon-nucleon interaction the one-boson exchange potentials Bonn A,B,C and for comparison the Walecka model are used. The T-matrix is determined from the Thompson equation and is projected onto five covariant amplitudes. By the restriction to positive energy states an ambiguity arises in the relativistic Brueckner approach which is discussed here in terms of the pseudo-scalar and the pseudo-vector projection. The influence of the coupling of the nucleon via the T-matrix as an effective two-nucleon interaction to the nuclear medium is expressed by the self-energy. In particular we investigate the scalar and vector components of the self-energy for the different one-boson exchange potentials and discuss their density and momentum dependence. We estimate the uncertainty of the self-energy due to the pseudo-scalar and the pseudo-vector choice. Usually the momentum dependence of the self-energy is thought to be weak, however, we find that this depends on the one-boson exchange potentials. For the Bonn potentials, in contrast to the $\sigma \omega-$ potential, the momentum dependence is strikingly strong above as well as below the Fermi surface. We compare with the results of other groups and study the effects on the equation of state and the nucleon optical potential.
\end{abstract}

21.30.+y, 21.65.+f, 24.10.Cn

\section{INTRODUCTION}

In the relativistic (or Dirac-) Brueckner model the dynamical two-nucleon correlations in infinite nuclear matter are studied. The formalism is based on an effective quantum field theory for mesons and nucleons [1] and thus ignores the underlying quark nature of the nucleon. In the Brueckner model the T-matrix (or Brueckner G-matrix) serves as an effective in-medium two-body interaction. It is determined by a self-consistent summation of the ladder diagrams in a quasipotential approximation (Thompson equation) to the Bethe-Salpeter equation, see, e.g., Ref. [2. The bare nucleon-nucleon (ladder) interaction is described by one-boson exchange potentials. In the present work we apply the Bonn potentials A,B,C of Refs. [3,9] and for comparison also a simple $\sigma \omega$-potential which is well known from the Walecka model [1]. The nucleon inside the nuclear medium is treated as a dressed Dirac particle where the influence of the coupling to the surrounding nucleons via the T-matrix serving as an effective interaction is expressed by the nucleon self-energy. The self-energy has a Lorentz structure with large scalar and four-vector components and modifies the single-particle spectrum and the spinor wave functions.

The relativistic Brueckner model has been developed in the last decades by various authors. At the beginnig of the 80's the relativistic problem was considered by the Brooklyn group [1,5] in first order perturbation theory. In the following the covariant formalism to a self-consistent treatment of the Thompson equation was developed by Horowitz and Serot [6] and is in detail outlined in Ref. [7]. However, in these works the nucleon-nucleon interactions was described within the framework of the $\sigma \omega$-model. Calculations with realistic nucleon-nucleon interactions have been performed by Brockmann and Machleidt [8,9] and later on by ter Haar and Malfliet [10,11]. A more rigorous derivation of the Brueckner approach in the framework of relativistic Green's function can be found in Ref. [12]. In the present work we refer to the formalism described in Refs. [7],10].

The main difference between the relativistic Brueckner approach of Refs. [6 11] and previous works is the use of medium dependent spinors and the requirement of self-consistency for both the spinor wave functions and the singleparticle spectrum. The Dirac spinors of the nucleon in the medium differ considerably from the free spinors due to the large scalar and vector self-energies. These introduce an additional density dependence into the spinor matrix elements of the one-boson exchange potentials and thus into the T-matrix. This dynamical effect is absent in nonrelativistics. Another new aspect of the modern Brueckner approach is that the inertial frames where the T-matrix is determined and where it is used have to be distinguished carefully. Due to the neglection of the retardation in the meson propagators the one-boson exchange potentials and the correspondingly T-matrix serve as quasi-potentials in the two-particle center-of-mass (c.m.) frame. Therefore the relativistic Thompson equation is solved in this system which can be done by numerical standard techniques, as, e.g., in the non-relativistic case of Ref. 13. On the other hand single-particle quantities like the self-energy of the nucleon are calculated in the nuclear matter rest frame, where the single-particle distribution, the Fermi sea, is naturally defined. Thus a covariant representation of the T-matrix is required to connect both frames. Even though, e.g., the single particle potential can be calculated with some 
additional approximations avoiding this representation, as done in Ref. [9], the covariant technique is unavoidable in order to calculate the self-energy components themselves.

In the relativistic Brueckner approach the self-consistent spinors are restricted to positive energy states. A different approach in the full Dirac space which is also based on one-boson exchange potentials is used in Refs. [4, 14]. But in a full treatment one should further include renormalisation effects like the vacuum fluctuation contributions to the self-energy, as discussed in the context of the relativistic Hartree approximation in Refs. [1],7], and solve the full BetheSalpeter equation self-consistently. However, then the one-boson exchange potentials would be inappropriate. But these are still outstanding problems. In the standard Brueckner model which we discuss in the present work one gets around the problem of renormalization. The masses and coupling constants in the model are physical renormalized quantities and as a consequence one neglects the effective negative-energy states. However, the effective positiveenergy spinors still contain admixtures of free negative-energy states because they are a superposition of free positiveand negative-energy spinors. To Lorentz transform the T-matrix as we have mentioned above the positive energy on-shell T-matrix is projected onto five invariant amplitudes. By the restriction to positive energy states an ambiguity arises [15] which is discussed in terms of the pseudo-scalar and the pseudo-vector representation of the T-matrix in the Dirac space. From analogy to the one-pion exchange coupling in the nucleon-nucleon potential the pseudo-vector choice is more natural and further it is known from meson theory analysis [16] that this choice suppresses the coupling to the negative-energy states. However, the difference between both choices also states a measure for the uncertainty concerning the determination of the self-energy components due to the restriction on positive energy spinors. One issue of the present work is to estimate this uncertainty.

Thus, the aim of this work is to determine the self-energy components, to estimate their uncertainty due to different covariant representations of the T-operator and different nucleon-nucleon potentials and to examine the sensitivity of some commonly used observables, i.e., the equation of state and the optical potential on the self-energies. We are investigating in detail the density and the momentum dependence of the scalar and the vector components and compare our results to those of other groups [9, [9, 10,17]. Thereby the momentum dependence can just be determined in a first approximation because it is neglected in the self-consistency treatment of the Brueckner model. Commonly this approximation is justified by the assumption of a weak momentum dependence, at least inside the Fermi sea, but there have been also indications that this assumption depends on the chosen one-boson exchange potential [15]. We show here for the first time the momentum dependence over its full range, i.e., below and above the Fermi surface, and we find it in the case of the Bonn A,B,C potentials of the order of several $100 \mathrm{MeV}$, i.e., to be strikingly strong. In difference to the most publications in the field we focus our discussion to the self-energy components themselves.

It is meanwhile well known that the nuclear matter saturation properties are much better reproduced in the relativistic than in the non-relativistic Brueckner approach, i.e., the predictions for the saturation point of a variety of nucleon-nucleon potentials are located along the Coester band which meets - in the relativistic case - the empirical area [9]. The same also holds, e.g., for the nucleon optical potential. However, as we also find in the present analysis these commonly studied observables are not really sensitive to the self-energy components since they mainly depend on a cancellation of the components. The situation changes in the application of the Brueckner theory beyond infinite nuclear matter. When applying relativistic Brueckner results to the description of finite nuclei, e.g., in the framework of an effective (density dependent) relativistic mean field theory [18] the self-energies start to play a decisive role. This fact is, however, most pronounced in the investigation of heavy ion collisions. Different from resting nuclear matter here the scalar and vector components are out of balance which is due to their different Lorentz transformation character and diverse observables are in particular sensitive to the magnitude of the fields [19]. Furthermore the momentum space configuration can be highly anisotropic and rather corresponds to two counterstreaming currents of nuclear matter which can be described by two Lorentz-elongated Fermi ellipsoids. Approximate determinations of relativistic Brueckner mean fields for such configurations have been performed in [20] and encouraging applications to heavy ion collisions in [21]. Thus the density and momentum dependence of the nuclear self-energy components as the quantity which most prominant characterizes the medium effects is a question of particular interest.

This paper is organized in the following way: in the next section we review the relativistic Brueckner approach. There are still some technical differences in the standard Brueckner model between Ref. [7] and Refs. [10 12]. Here we follow the approach of Horowitz and Serot [1] which allows the use of standard techniques for the solution of the Thompson equation known from earlier works [13,22]. However, we use the modern one-boson exchange potentials, treat the isospin channels and also the real and imaginary part of the T-matrix separately and project on both, the pseudo-scalar and the pseudo-vector representation. In the following section we discuss our numerical results. The calculations with the $\sigma \omega$-potential are compared with results from Ref. [7]. We further discuss the density and momentum dependence of the scalar and vector self-energy, the influence of pseudo-scalar and pseudo-vector choice is analyzed and we compare to the results of Brockmann and Machleidt [9] and the Groningen group [10, 17]. Further the equation of state and the optical potential are shown and their sensitivity to the self-energy components is analyzed. In the final section a summary is given. 


\section{RELATIVISTIC BRUECKNER APPROACH}

In the relativistic Brueckner approach the nucleon inside the nuclear medium may be viewed as a dressed particle in conseqeunce of its two-body interaction with the surrounding nucleons. This problem is stated as a coupled set of three non-linear integral equations

$$
\begin{aligned}
& T=V+i \int V Q G G T, \\
& G=G^{\circ}+G^{\circ} \Sigma G, \\
& \Sigma=-i \int_{F}(\operatorname{Tr}[G T]-G T) .
\end{aligned}
$$

The T-matrix is calculated in the ladder approximation of the Bethe-Salpeter equation (11) and the bare nucleonnucleon interaction is described by one-boson exchange potentials $V$. The intermediate nucleons are given by the two-body propagator $i G G$ which is, however, usually replaced by an effective propagator [2], i.e., the BlankenbeclerSugar propagator or the Thompson propagator. In the present work the Thompson choice is applied. The Pauli operator $Q$ accounts for the influence of the medium by the Pauli principle and projects the intermediate scattering states outside the Fermi sea. The dressed one-body Green propagator $G$ is calculated via the Dyson equation (2) from the free propagator $G^{\circ}$. The influence of the coupling to the surrounding nucleons is expressed by the selfenergy $\Sigma$ of the nucleon. In the Brueckner formalism this self-energy is determined by summing up the direct and exchange interactions with all the nucleons inside the Fermi sea $F$, see Eq. (3). Here the T-matrix plays the role of an effective medium dependent two-body interaction. In the relativistic Brueckner approach one introduces additional approximations for solving the equations (1-3) which are sketched in the following, for further reading see, e.g., Refs. [7, 10 .

\section{A. Self-consistent spinor basis}

The most general form of the Lorentz-structure of the self-energy compatible with translational invariance, hermiticity, parity conservation, time-reversal invariance and rotational invariance (in the nuclear matter rest frame) is

$$
\Sigma(k)=\Sigma_{s}(k)-\Sigma^{\mu}(k) \gamma_{\mu}=\Sigma_{s}(k)-\gamma_{0} \Sigma_{0}(k)+\gamma \cdot \mathbf{k} \Sigma_{v}(k)
$$

with a scalar part $\Sigma_{s}$ and a vector part $\Sigma^{\mu}=\left(\Sigma_{0}, \mathbf{k} \Sigma_{v}\right)$. Due to the Dyson equation (2) the full Green function has the formal solution

$$
G^{-1}=G^{\circ-1}-\Sigma(k)=\gamma_{\mu} k^{* \mu}-M^{*}(k)-i \operatorname{Im}[\Sigma(k)]
$$

where we have introduced the effective mass and the kinetic momentum

$$
M^{*}(k)=M+\operatorname{Re} \Sigma_{s}(k), \quad k^{* \mu}=k^{\mu}+\operatorname{Re} \Sigma^{\mu}(k) .
$$

Re and Im denote real and imaginary part since (above the Fermi surface) the self-energy in general will be complex. Here we adopt the quasiparticle approximation [12, i.e., the Im [ $\Sigma]$ will be neglected in Eq. (5). This means that the decay width of the dressed nucleon is set equal to zero, resulting in an infinite lifetime of this 'quasiparticle'. In the relativistic Brueckner approach the full Green function $G$ is replaced by its positive energy part. Thus the self-energy in Eq. (3) is determined by the part proportional to the Fermi sea $F$, the so-called Dirac Green function $G_{D}$ [1:7]

$$
G_{D}(k)=\left[\gamma_{\mu} k^{* \mu}+M^{*}(k)\right] 2 \pi i \delta\left(k^{* 2}-M^{* 2}(k)\right) \Theta\left(k^{* 0}\right) \Theta\left(k_{F}-|\mathbf{k}|\right)
$$

which eliminates the divergent contributions from the negative energy sea. However, in the form of Eq. (7) $G_{D}$ is restricted to the description of nuclear matter at rest. In order to achieve general convenience the Fermi sphere $\Theta\left(k_{F}-|\mathbf{k}|\right)$ has to be replaced by an Fermi ellipsoid $\Theta\left(E_{F}-k^{* \mu} u_{\mu}\right)$ with the Fermi energy $E_{F}=\sqrt{\left(1+\Sigma_{v}\right)^{2} k_{F}^{2}+M^{* 2}}$ and the boost 4 -velocity $u^{\mu}=(\gamma, \mathbf{u} \gamma)$ [20]. In Eq. (7) the momenta of the quasiparticles are put on mass-shell, which is expressed by the $\delta$-distribution and thus $k^{* 0}=E^{*}(\mathbf{k})=\sqrt{\mathbf{k}^{* 2}+M^{* 2}}$. Hence the self-energy $\Sigma(k)=\Sigma\left(|\mathbf{k}|, k_{F}\right)$ depends only on the three-momentum $|\mathbf{k}|$ and, of course, on the respective density or $k_{F}$. 
Furthermore, in the relativistic Brueckner model one applies a mean field approximation to $\Sigma$, Eq. (4), which allows a simple formulation of the self-consistency problem. The explicit momentum dependence of the self-energy which enters via the term $\mathbf{k} \Sigma_{v}$ can be dealt with by introducing the reduced kinetic momentum $\tilde{k}^{* \mu}=k^{* \mu} /\left(1+\Sigma_{v}\right)$ and the reduced effective mass $\tilde{M}^{*}=M^{*} /\left(1+\Sigma_{v}\right)$. Further one neglects the momentum dependence of the effective mass $\left(M^{*}\right.$ or $\left.\tilde{M}^{*}\right)$. Thus, the nucleons fulfill a quasi-free Dirac equation

$$
\left[\gamma_{\mu} \tilde{k}^{* \mu}-\tilde{M}^{*}\right] u_{\lambda}(k)=0
$$

and using the normalization of Ref. [23] the self-consistent positive-energy spinors of helicity $\lambda$ are defined as

$$
u_{\lambda}(k)=\sqrt{\frac{\tilde{E}^{*}(k)+\tilde{M}^{*}}{2 \tilde{M}^{*}}}\left(\begin{array}{c}
1 \\
\frac{2 \lambda|\mathbf{k}|}{\tilde{E}^{*}(k)+\tilde{M}^{*}}
\end{array}\right) \chi_{\lambda}
$$

with $\chi_{\lambda}$ being a Pauli spinor. The Dirac spinors depend on the effective mass and thus on the nuclear density. The matrix-elements of the T-matrix and the one-boson exchange potentials $V$, Eq. (11), are calculated with these spinors. In contrast to non-relativistic Brueckner theory this fact introduces an additional density dependence into the interaction which is one major reason for the great success of the relativistic treatment. This density dependence is mediated by the additional parameter $\tilde{M}^{*}$ which is fixed at a reference point, usually at $|\mathbf{k}|=k_{F}$. $\tilde{M}^{*}$ is obtained as the solution of the non-linear equation (reference spectrum approximation)

$$
\tilde{M}^{*}=M+\Sigma_{s}\left(k_{F}, \tilde{M}^{*}\right)-\tilde{M}^{*} \Sigma_{v}\left(k_{F}, \tilde{M}^{*}\right)
$$

which follows from the definition given above. Self-consistency is achieved by determining for a start value of $\tilde{M}^{*}$ the medium-dependent spinors of Eq. (9), next the T-matrix from Eq. (11) and finally the self-energy and the new $\tilde{M}^{*}$. This procedure is repeated until convergence is reached.

\section{B. Covariant T-matrix amplitudes}

The T-matrix, in Eq. (11), is most easily determined in the two-particle center-of-mass (c.m.) system while the self-energy, Eq. (3), is calculated in the nuclear matter rest frame. Therefore we will determine in a second step covariant amplitudes of the T-matrix. The Thompson propagator (and similar the Blankenbecler sugar propagator) projects the intermediate nucleons onto positive energy states and restricts the exchanged energy transfer by $\delta\left(k^{0}\right)$ to zero. Thus equation (1) is reduced to a three dimensional integral equation of the Lippmann-Schwinger type, the so called Thompson equation [2, 10 ]

$$
\left.T(\mathbf{p}, \mathbf{q}, x)\right|_{\mathrm{c} . \mathrm{m} .}=V(\mathbf{p}, \mathbf{q})+\int \frac{d^{3} \mathbf{k}}{(2 \pi)^{3}} V(\mathbf{p}, \mathbf{k}) \frac{M^{* 2}}{E^{* 2}(\mathbf{k})} \frac{Q(\mathbf{k}, x)}{2 E^{*}(\mathbf{q})-2 E^{*}(\mathbf{k})+i \epsilon} T(\mathbf{k}, \mathbf{q}, x) .
$$

Here the Thompson equation is given in the two-nucleon c.m.-frame where $\mathbf{p}=\left(\mathbf{p}_{1}-\mathbf{p}_{2}\right) / 2=\left(\mathbf{p}_{1}^{*}-\mathbf{p}_{2}^{*}\right) / 2$ is the relative momentum of the final states and similar $\mathbf{q}, \mathbf{k}$ are the relative momenta of the initial and intermediate states, respectively. The Thompson propagator and similar the Blankenbecler-Sugar propagator imply that the timelike component of the momentum transfer in $V$ and $T$ is set equal to zero which is a natural constraint in the c.m.frame, however, not a covariant one. Hence we solve the Thompson equation in the c.m.-frame in contrast to, e.g., the approach of Ref. [9]. The starting energy in Eq. (11) is already fixed: $\sqrt{s^{*}}=2 E^{*}(\mathbf{q})$. The Pauli operator $Q$ explicitely depends on the chosen frame, i.e., on the boost 3-velocity $\mathbf{u}$ into the c.m.-frame. With $x$ we denote the set of parameters $x=\left\{k_{F}, M^{*},|\mathbf{u}|\right\}$ on which the T-matrix actually depends.

We solve the Thompson equation (11) for the on-shell T-matrix $(|\mathbf{p}|=|\mathbf{q}|)$ in the c.m.-system and thereby apply standard techniques which are described in detail by Erkelenz [22]. We construct the positive-energy helicity Tmatrix elements from the $J M L S>$-scheme. In the on-shell case only five of the sixteen helicity matrix elements are independent which follows from general symmetries, see Ref. [22]. After a partial wave projection onto the $\mid J M L S>-$ states the integral reduces to a one-dimensional integral over the relative momentum $|\mathbf{k}|$ and Eq. (11) decouples into three subsystems of integral equations for the uncoupled spin singlet, the uncoupled spin triplet and the coupled

${ }^{1}$ From now on we omit the tilde because in the following we normally deal with $\tilde{M}^{*}, \tilde{k}^{* \mu}$. 
triplet states. The Pauli operator $Q$ is replaced by an angle averaged Pauli operator $\bar{Q}$ [7]. Since the Fermi sphere is deformed to a Fermi ellipsoid in the two-nucleon c.m.-frame $\bar{Q}$ is evaluated for such a Fermi-ellipsoid.

$$
\bar{Q}(|\mathbf{k}|, x)= \begin{cases}0 & |\mathbf{k}|<k_{-} \\ \left(\gamma E^{*}(\mathbf{k})-E_{F}\right) / u \gamma|\mathbf{k}| \text { for } & k_{-} \leq|\mathbf{k}| \leq k_{+} \\ 1 & |\mathbf{k}|>k_{+}\end{cases}
$$

with $k_{-}=\sqrt{k_{F}^{2}-u^{2} E_{F}^{2}}, k_{+}=\gamma\left(u E_{F}+k_{F}\right)$ and $u=|\mathbf{u}|$. We are solving the integral equations by the matrix inversion techniques of Haftel and Tabakin [13. Real and imaginary parts of the T-matrix are calculated separately by the principal-value treatment given in Ref. 24]. Due to the antisymmetry of the two-fermion states we can restore the total isospin $\mathrm{I}$ of the two-nucleon system $(\mathrm{I}=0,1)$ with the help of the selection rule: $(-)^{L+S+\mathrm{I}}=-1$. From the five independent on-shell amplitudes in the $\mid J M L S>$-representation the five independent partial wave amplitudes in the helicity representation (for $\mathrm{I}=0,1$ and real and imaginary part separately) are obtained by inversion of Eq. (3.32) and then of Eq. (3.28) of Ref. [22]. The summation over the total angular momentum $J$ (we have taken $J \leq 6$ and for comparison $J \leq 12$ ) yields the full helicity matrix element

$$
\sum_{J}\left[\frac{2 J+1}{4 \pi}\right] d_{\lambda \lambda^{\prime}}^{J}(\theta)<|\mathbf{p}| \lambda_{1}^{\prime} \lambda_{2}^{\prime}\left|T^{J, \mathrm{I}}(x)\right||\mathbf{q}| \lambda_{1} \lambda_{2}>=<\mathbf{p} \lambda_{1}^{\prime} \lambda_{2}^{\prime} \mathrm{II}_{3}|T(x)| \mathbf{q} \lambda_{1} \lambda_{2} \mathrm{II}_{3}>.
$$

Here $\theta$ is the scattering angle between $\mathbf{q}$ and $\mathbf{p}$ and $\lambda=\lambda_{1}-\lambda_{2}, \lambda^{\prime}=\lambda_{1}^{\prime}-\lambda_{2}^{\prime}$. The reduced rotation matrices $d_{\lambda \lambda^{\prime}}^{J}(\theta)$ are those defined by Rose [25]. The matrix element (13) is actually independent of the third component of the isospin $\mathrm{I}_{3}$. The next step is the projection of the five independent helicity amplitudes onto five covariant amplitudes. Therefore the T-operator is expanded into the basis matrices in the Dirac spinor space

$$
T^{\mathrm{I}}=T^{\mathrm{S}, \mathrm{I}} 1_{(1)} 1_{(2)}+T^{\mathrm{V}, \mathrm{I}} \gamma_{(1)}^{\mu} \gamma_{(2) \mu}+T^{\mathrm{T}, \mathrm{I}} \sigma_{(1)}^{\mu \nu} \sigma_{(2) \mu \nu}+T^{\mathrm{P}, \mathrm{I}}\left(\frac{\not K \gamma_{5}}{2 M^{*}}\right)_{(1)}\left(\frac{\not K \gamma_{5}}{2 M^{*}}\right)_{(2)}+T^{\mathrm{A}, \mathrm{I}}\left(\gamma_{5} \gamma^{\mu}\right)_{(1)}\left(\gamma_{5} \gamma_{\mu}\right)_{(2)}
$$

The subscripts (1),(2) denote the particle on which the matrix acts. This expansion, however, is not unique since we have specified the Dirac matrix structure of the $\mathrm{T}$ operator by its action only on positive energy states. The expansion of Eq. (14) is given with $T^{\mathrm{P}, \mathrm{I}}$ a pseudo-vector interaction $\mathbb{K} \gamma_{5}$. It is defined as $\mathbb{K}=\gamma^{\mu} \mathrm{K}_{\mu}$ with the transferred momentum at the vertex $\mathrm{K}^{\mu}=p_{f}^{\mu}-p_{i}^{\mu}=p_{f}^{* \mu}-p_{i}^{* \mu}$ where $f, i$ indicates final and initial momentum. We will, however, also adopt a pseudo-scalar interaction where the fourth term in Eq. (14) is replaced by $T^{\mathrm{P}, \mathrm{I}} \gamma_{5}^{(1)} \gamma_{5}^{(2)}$ as it was also done by Horowitz and Serot [7]. The pseudo-vector choice is, e.g., made by the Groningen group. This choice is supported by the argument that it agrees with the one-pion exchange coupling in the nucleon-nucleon potential and furthermore suppresses the coupling to the negative energy states which are neglected in the Brueckner approach [10,16]. In the present work we apply both variants since we want to examine the influence of the different choices on the self-energy.

Next we take the helicity matrix elements of Eq. (14). On the r.h.s. the helicity states are acting directly on the matrix operators which are now abbreviated by $\kappa_{m}^{(i)}$ for $\mathrm{i}=1,2$ and $m \varepsilon\{S, V, T, P, A\}$

$$
<\mathbf{p} \lambda_{1}^{\prime} \lambda_{2}^{\prime} \mathrm{II}_{3}|T(x)| \mathbf{q} \lambda_{1} \lambda_{2} \mathrm{II}_{3}>=\sum_{m}<\mathbf{p} \lambda_{1}^{\prime} \lambda_{2}^{\prime}\left|\kappa_{m}^{(1)} \kappa_{m}^{(2)}\right| \mathbf{q} \lambda_{1} \lambda_{2}>T^{m, \mathrm{I}}(|\mathbf{p}|, \theta, x)
$$

The helicity matrix elements on the r.h.s. are given explicitely, e.g., in Eqs. (2.11-2.17) of Ref. [22]. For onshell scattering between positive energy states the matrix elements of the pseudo-scalar (PS) and the pseudo-vector (PV) matrix operators are identical [16]. This vertex equivalance follows immediately from the Dirac Eq. (8): $\bar{u}(p)\left(\frac{p^{*}-q^{*}}{2 M^{*}}\right) \gamma_{5} u(q)=\bar{u}(p) \gamma_{5} u(q)$. Thus the covariant amplitudes $T^{\mathrm{PS}}=T^{\mathrm{PV}} \equiv T^{\mathrm{P}}$ are identical as well. The difference between both representations of the T-matrix in Dirac space enters only at the one-body level when calculating the self-energy [26]. For fixed parameters $(x,|\mathbf{p}|=|\mathbf{q}|, \theta)$ equation (15) is a matrix relation between the five independent helicity amplitudes $T_{i}^{\mathrm{I}}$ (five of the sixteen amplitudes numbered with $i=\left\{\lambda_{1}^{\prime} \lambda_{2}^{\prime} ; \lambda_{1} \lambda_{2}\right\}=1, . ., 5$ ) on the l.h.s. and the five unknown covariant amplitudes $T^{m, I}$

$$
T_{i}^{\mathrm{I}}=\frac{1}{M^{* 2}} \sum_{m} C_{i m} T^{m, \mathrm{I}}
$$

This corresponds to Eq. (3.23) of Ref. [7] with respect to our different normalization of the spinor basis, Eq. (9). The covariant amplitudes are determined by a matrix inversion of equation (16). This, however, only has to be done for 
two angles since for on-shell scattering in the two-particle c.m.-frame only two scattering angles appear, i.e., $\theta=0$ for the direct and $\theta=\pi$ for the exchange interaction. However, now the matrix becomes singular because two of the five helicity amplitudes vanish. Therefore it is useful to extract the leading angular dependence of the matrix $C_{i m}$ as well as of the helicity amplitudes $T_{i}^{\mathrm{I}}$ from the rotation matrices $d_{\lambda \lambda^{\prime}}^{J}(\theta=0, \pi)$ in Eq. (13). Thus, the limit $\theta \rightarrow 0, \pi$ can be performed analytically. This method has been developed in appendix $\mathrm{C}$ of Ref. 阿 and formula (16) can be found there for the limiting angles $0, \pi$ (Eqs. (C.10,11)). Proceeding this way we determine the real and the imaginary part of the five covariant amplitudes for the direct and exchange contribution in both, the isospin singlet and triplet channels of the T-matrix $T^{m, \mathrm{I}=0,1}(\theta=0, \pi)$.

\section{Self-energy components}

Now we are able to calculate the self-energy. Inserting the Green function, Eq. (7), and the Dirac representation of the T-matrix, Eq. (14), into the definition of the self-energy, Eq. (3), yields

$$
\begin{aligned}
\Sigma_{\alpha \beta} & =\int \frac{d^{3} \mathbf{q}}{(2 \pi)^{3}} \frac{\Theta\left(k_{F}-|\mathbf{q}|\right)}{4 E^{*}(\mathbf{q})}\left\{M^{*} 1_{\alpha \beta}\left[4 T_{D}^{\mathrm{S}}-T_{X}^{\mathrm{S}}-4 T_{X}^{\mathrm{V}}-12 T_{X}^{\mathrm{T}}+4 T_{X}^{\mathrm{A}}-\frac{\left(k^{* \mu}-q^{* \mu}\right)^{2}}{4 M^{* 2}} T_{X}^{\mathrm{P}}\right]\right. \\
& \left.+\not_{\alpha \beta}^{*}\left[4 T_{D}^{\mathrm{V}}-T_{X}^{\mathrm{S}}+2 T_{X}^{\mathrm{V}}+2 T_{X}^{\mathrm{A}}-\frac{\left(k^{* \mu}-q^{* \mu}\right)^{2}}{4 M^{* 2}} T_{X}^{\mathrm{P}}\right]-\left(\not q^{*}-\not k^{*}\right)_{\alpha \beta} \frac{2 q_{\mu}^{*}\left(k^{* \mu}-q^{* \mu}\right)}{4 M^{* 2}} T_{X}^{\mathrm{P}}\right\}
\end{aligned}
$$

where the $\alpha, \beta$ denote both, Dirac (spin) and isospin indices. The direct and exchange parts of the T-matrix denoted by $D$ and $X$ are calculated from isospin sums of the isospin projection operators which yields $T_{D}^{m}=T_{D}^{m \mathrm{I}=0}+3 T_{D}^{m \mathrm{I}=1}$ and $T_{X}^{m}=-T_{X}^{m \mathrm{I}=0}+3 T_{X}^{m \mathrm{I}=1}$. The self-energy as a one-body operator in spinor space posseses components proportional to the unity matrix 1 and to $\gamma^{\mu}$ as postulated in equation (4). From this relation the self-energy components $\Sigma_{s, 0, v}$ follow by taking traces

$$
\begin{aligned}
\Sigma_{s}\left(|\mathbf{k}| ; M^{*}, k_{F}\right) & =\frac{1}{8} \operatorname{Tr}[\Sigma]=\int \frac{d^{3} \mathbf{q}}{(2 \pi)^{3}} \Theta\left(k_{F}-|\mathbf{q}|\right) \frac{M^{*}}{E^{*}(\mathbf{q})} T_{s}(\mathbf{k}, \mathbf{q} ; x), \\
\Sigma_{0}\left(|\mathbf{k}| ; M^{*}, k_{F}\right) & =-\frac{1}{8} \operatorname{Tr}\left[\gamma^{0} \Sigma\right]=\int \frac{d^{3} \mathbf{q}}{(2 \pi)^{3}} \Theta\left(k_{F}-|\mathbf{q}|\right) T_{(0)}(\mathbf{k}, \mathbf{q} ; x), \\
\Sigma_{v}\left(|\mathbf{k}| ; M^{*}, k_{F}\right) & =-\frac{1}{8} \operatorname{Tr}[\gamma \cdot \mathbf{k} \Sigma]=\int \frac{d^{3} \mathbf{q}}{(2 \pi)^{3}} \Theta\left(k_{F}-|\mathbf{q}|\right) \frac{\mathbf{q}^{*} \cdot \mathbf{k}}{|\mathbf{k}|^{2} E^{*}(\mathbf{q})} T_{v}(\mathbf{k}, \mathbf{q} ; x)
\end{aligned}
$$

where we have used the abbreviations

$$
\begin{gathered}
T_{s}(\mathbf{k}, \mathbf{q} ; x)=\frac{1}{4}\left[4 T_{D}^{\mathrm{S}}-T_{X}^{\mathrm{S}}-4 T_{X}^{\mathrm{V}}-12 T_{X}^{\mathrm{T}}+4 T_{X}^{\mathrm{A}}+\frac{k_{\mu}^{*} q^{* \mu}-M^{* 2}}{2 M^{* 2}} T_{X}^{\mathrm{P}}\right], \\
T_{(0)}(\mathbf{k}, \mathbf{q} ; x)=\frac{1}{4}\left[-4 T_{D}^{\mathrm{V}}+T_{X}^{\mathrm{S}}-2 T_{X}^{\mathrm{V}}-2 T_{X}^{\mathrm{A}}+\frac{E^{*}(\mathbf{k})}{E^{*}(\mathbf{q})} \frac{M^{* 2}-k_{\mu}^{*} q^{* \mu}}{2 M^{* 2}} T_{X}^{\mathrm{P}}\right], \\
T_{v}(\mathbf{k}, \mathbf{q} ; x)=\frac{1}{4}\left[-4 T_{D}^{\mathrm{V}}+T_{X}^{\mathrm{S}}-2 T_{X}^{\mathrm{V}}-2 T_{X}^{\mathrm{A}}+\frac{|\mathbf{k}|}{q_{z}} \frac{M^{* 2}-k_{\mu}^{*} q^{* \mu}}{2 M^{* 2}} T_{X}^{\mathrm{P}}\right] .
\end{gathered}
$$

In the pseudo-scalar case [7] all the cofactors of $T_{X}^{\mathrm{P}}$ are replaced just by -1 which yields $T_{(0)}=T_{v}$. The self-energy of a nucleon is calculated in the nuclear matter rest frame by integrating the effective two-body interaction over the Fermi sea. The 3 -momenta $\mathbf{k}$, $\mathbf{q}$ of a nucleon pair are given in this frame and we define $\mathbf{P}^{*}=\mathbf{k}+\mathbf{q}=\mathbf{k}^{*}+\mathbf{q}^{*}$. On the other hand the covariant amplitudes are determined in the two-nucleon c.m. system and thus depend on the respective quantities in that frame

$$
\begin{aligned}
& T_{D}^{m}(\mathbf{k q}, \mathbf{k q} ; x)=\quad T^{m \mathrm{I}=0}\left(\left|\mathbf{p}_{\mathrm{c} . \mathrm{m} .}\right|, \theta=0, x\right)+3 T^{m \mathrm{I}=1}\left(\left|\mathbf{p}_{\mathrm{c} . \mathrm{m} .}\right|, \theta=0, x\right) \\
& T_{X}^{m}(\mathbf{k q}, \mathbf{q k} ; x)=-T^{m \mathrm{I}=0}\left(\left|\mathbf{p}_{\mathrm{c} . \mathrm{m} .}\right|, \theta=\pi, x\right)+3 T^{m \mathrm{I}=1}\left(\left|\mathbf{p}_{\mathrm{c} . \mathrm{m} .}\right|, \theta=\pi, x\right)
\end{aligned}
$$

From the invariant mass $s^{*}=\left(E^{*}(\mathbf{k})+E^{*}(\mathbf{q})\right)^{2}-\mathbf{P}^{* 2}$, the relative momentum in the c.m.-frame follows by $\left|\mathbf{p}_{\mathrm{c} . \mathrm{m} .}\right|=$ $\sqrt{s^{*} / 4-M^{* 2}}$. As already mentioned above, $x$ abbreviates the set of parameters $x=\left\{k_{F}, M^{*},|\mathbf{u}|\right\}$, where the boost 3 -velocity (from the nuclear matter into the c.m.-frame) is now given by $\mathbf{u}=\mathbf{P}^{*} / \sqrt{s^{*}+\mathbf{P}^{* 2}}$. In the two-particle 
c.m.-frame there exist only two possible scattering angles, i.e., $\theta=0$ for the direct and $\theta=\pi$ for the exchange amplitude. Numerically we solve the Thompson equation (11) and determine the covariant amplitudes $T^{m, I}$ for a suitable two-dimensional range of the quantities $\mathbf{p}_{c . m .},|\mathbf{u}|$. Using the azimuthal symmetry we calculate the selfenergy, Eqs. (18-20), by a two-dimensional integral over the Fermi sphere. Therefore for each nucleon pair we have to interpolate the covariant amplitudes at the respective values of $\mathbf{p}_{\text {c.m. }}$. and $|\mathbf{u}|$. The resulting self-energy, taken at the Fermi surface $|\mathbf{k}|=k_{F}$, yields the new value of the effective mass $M^{*}$, Eq. (10). This value serves as the input for the next iteration. This procedure is repeated until a convergence of the effective mass is achieved.

\section{NUCLEAR MATTER RESULTS}

For the solution of the Thompson equation (11) we apply the Bonn A,B,C potentials of Refs. [3, 9] as the nucleonnucleon interaction $V$. The potentials are calculated with the OBNNS code of R. Machleidt [27]. The Bonn potentials are based on the exchange of the six nonstrange bosons with masses below $1 \mathrm{GeV}(\pi, \eta, \rho, \omega, \delta, \sigma)$. For the pion a derivative pseudovector coupling is applied. The three parametrizations A,B and $\mathrm{C}$ differ essential in the $\pi \mathrm{NN}$ formfactor and as a consequence in the strength of the nuclear tensor force. Actually we find only minor differences of a few $\mathrm{MeV}$ in the resulting self-energies, see below, and thus we present mainly the Bonn C results. For comparison with the results of Horowitz and Serot we also employ the simple $\sigma \omega$-meson exchange of Ref. [7] with $g_{\sigma}^{2}=91.64, g_{\omega}^{2}=136.2$ and use the form-factor given therein.

\section{A. self-energy components}

\section{1. density dependence}

First we want to demonstrate the reliability of our approach by comparison with the results of Ref. [7]. In Fig. 1 we show the self-consistent effective mass $M^{*}$, Eq. (10), as a function of the Fermi momentum $k_{F}$ which is a measure for the density $\varrho=2 /\left(3 \pi^{2}\right) k_{F}^{3}$. Our results with the $\sigma \omega$-meson exchange potential and the pseudo-scalar decomposition of the T-matrix reproduce quite well the values taken from Ref. [7]. The slight deviation for densities above saturation density (here $k_{F}=1.42 \mathrm{fm}^{-1}$ ) is partly due to the different effective two-nucleon propagators. As mentioned above, we are using the Thompson propagator, Eq. (11), while in Ref. [7] the Blankenbecler-Sugar propagator is applied. Furthermore, in Ref. [7] an additional approximation has been made. As discussed above, we are solving the Thompson equation for a two-dimensional grid of the relative momentum $|\mathbf{p}|$ and the c.m.-momentum $|\mathbf{P}|$ respectively the boostvelocity $|\mathbf{u}|$ and use this T-matrix for the determination of $\Sigma$. In Ref. 7 the $|\mathbf{P}|$-dependence is replaced by an "average $|\mathbf{P}|$ approximation" for each $|\mathbf{p}|$ which causes deviations mainly at high density systems. For the Bonn C potential the effective mass is significantly different from that of the $\sigma \omega$-model. Here we applied the pseudo-vector choice.

In Fig. 2 we show the density dependence of the self-energy, i.e., the scalar part $\Sigma_{s}$ as well as the time-like $-\Sigma_{0}$ and the space-like component $-k_{F} \Sigma_{v}$ of the vector self-energy. The self-energies are determined for the three Bonn potentials A,B,C and again we the pseudo-vector choice is applied. For the sake of a better comparison the sign of the vector self-energies is inversed and the dimensionless space-like component $\Sigma_{v}$ is multiplied by $k_{F}$. The scalar and also the vector self-energy component $\Sigma_{s},-\Sigma_{0}$ differ only slightly for the three Bonn potentials. The difference amounts about $5 \mathrm{MeV}$ at saturation density (here $k_{F}=1.35 \mathrm{fm}^{-1}$ ) and is still less than $10 \mathrm{MeV}$ at $k_{F}=1.90 \mathrm{fm}^{-1}$. In absolute magnitude Bonn $\mathrm{C}$ yields the weakest and Bonn $\mathrm{A}$ the strongest self-energies. The space-like vector self-energy $-k_{F} \Sigma_{v}$ is equal within line-width for the three Bonn potentials and is small in comparison to $\Sigma_{s}, \Sigma_{0}$, e.g., about $7 \%$ of $\Sigma_{0}$ at saturation density. For further analysis we therefore concentrate on the large components $\Sigma_{s}, \Sigma_{0}$ calculated with the Bonn $\mathrm{C}$ potential.

\section{2. pseudo-scalar versus pseudo-vector choice}

The two different representations of the T-matrix, i.e., the pseudo-scalar and the pseudo-vector case, represent an inherent ambiguity. This is, however, a common feature of all relativistic Brueckner calculations which specify the Dirac matrix structure of the T-matrix by using only positive energy T-matrix elements. In order to clarify the influence of this ambiguity in Figs. 3 . and 4 . we show the self-energy components $\Sigma_{s, 0}$ calculated exemplaryly with the Bonn C potential for both choices. In the pseudo-scalar case, see Fig. 3, the self-energies are larger by about 40-80 $\mathrm{MeV}$ (decreasing with increasing density) than in the pseudo-vector case (Fig. 4). This difference may be interpreted 
as a measure of the general uncertainty of the self-energy components arising from any particular projection of the Toperator on positive energy matrix elements only. This uncertainty is larger at low densities. To get more insight into the origin of this difference we decompose the self-energy in the two contributions stemming from the direct and the exchange T-amplitude respectively. E.g., in the pseudo-scalar case the direct part of the self-energy $\Sigma_{s}$ is determined by the integral over $T_{D}^{\mathrm{S}}$ while the exchange part is given by the integral over the sum of all exchange-amplitudes $-1 / 4 T_{X}^{\mathrm{S}}-T_{X}^{\mathrm{V}}-3 T_{X}^{\mathrm{T}}+T_{X}^{\mathrm{A}}-1 / 4 T_{X}^{\mathrm{P}}$, see Eq. (21) and the subsequent comment. We find that in the pseudo-scalar case direct and exchange parts of $\Sigma_{s, 0}$ are identical within less than $0.5 \mathrm{MeV}$, see Fig. 3. However, in the pseudo-vector case the factor accompanying the $T_{X}^{\mathrm{P}}$ amplitude in Eqs. (21) 22 reduces the exchange part by roughly $50 \%$. For comparison in the $\sigma \omega$-model, which lacks of pion exchange, the $T_{X}^{\mathrm{P}}$ amplitude is generally smaller and the effect, i.e., the reduction of the exchange part, amounts to roughly 25\%. Of course also the direct part is different from that of the pseudo-scalar case since we consider the self-consistently determined fields. We conclude that the pseudo-vector choice in general reduces the total self-energy which is due to a considerable reduction of the exchange contributions while the direct contributions are even enhanced.

In Fig. 5 we compare our results for $\Sigma_{s},-\Sigma_{0}$ obtained with the pseudo-vector choice and the Bonn $\mathrm{C}$ potential with the results of two other groups, i.e., the Groningen group [10] and the approach of Brockmann and Machleidt [9]. In Ref. [9] the relativistic Thompson equation is solved by standard methods [13] directly in the nuclear matter rest frame instead of the two-particle c.m.-frame. Therefore the Thompson Eq. (11) is Galilei transformed to the rest frame with the c.m.-momentum $\mathbf{P} / 2$. The relativistic single particle potential $U$ in the nuclear matter rest frame can be evaluated from this T-matrix by

$$
U(|\mathbf{k}|)=\frac{M^{*}}{E^{*}(\mathbf{k})} \operatorname{Re}<k|\Sigma| k>=\sum_{q \varepsilon F} \frac{M^{* 2}}{E^{*}(\mathbf{k}) E^{*}(\mathbf{q})} \operatorname{Re}<k q|T| k q-q k>
$$

with the definition of $\Sigma(|\mathbf{k}|)$ as in Eq. (3). The advantage of this method is that one is not forced to Lorentz transform the matrix elements between the nuclear matter rest frame and the two-particle c.m.-frame and thus a projection onto covariant amplitudes is obsolete which simplifies the task considerably. However, now the self-energy components $\Sigma_{s},-\Sigma_{0}$ cannot be calculated directly as in the projection method of Eqs. 18-20). Instead, one has to extract them from the single particle potential $U$. With the form of $\Sigma$ as in Eq. (14), however, thereby neglecting $\Sigma_{v}$ one finds

$$
U(|\mathbf{k}|)=\frac{M^{*}}{E^{*}(\mathbf{k})} \Sigma_{s}-\Sigma_{0}
$$

i.e., the single particle potential results from a cancellation of the huge scalar and vector components. Using a mean field ansatz for $\Sigma_{s, 0}$, i.e., neglecting their momentum dependence, one is able to determine them by a fit procedure to $U(|\mathbf{k}|)$. In Ref. [9] the self-energy components are constructed by this potential-fit method. In Fig. 5 we compare these results with our calculations both for the Bonn $\mathrm{C}$ potential. In the density range considered the fitted self-energies are generally smaller in absolute values by 50 up to more than $150 \mathrm{MeV}$, e.g., at saturation density they are reduced by about $75 \mathrm{MeV}$. In spite of this discrepancy both calculations have several features in common. E.g., the single particle potential, defined in Eq. (26), is almost identical and as we will see later on the same holds for the equation of state. Concerning these two quantities the main difference between both treatments lies in the reference frame where the T-matrix is actually calculated while the projection method on covariant amplitudes is not essential. At moderate densities the T-matrix depends only weakly on the c.m.-momentum $\mathbf{P}$ and respectively on the chosen frame. Thus the numerical results show only minor differences. Another feature in common is that also the potential-fit method yields self-energies for the three types of Bonn potentials which are equal within a few $\mathrm{MeV}$ [9].

In Fig. 5 we further compare to the results of the Groningen group [10]. They also perform a full relativistic Brueckner calculation solving the Thompson equation in the two-particle c.m.-system and using a projection of the T-matrix onto a pseudo-vector representation. The difference to our method is on the one hand the application of a different one-boson exchange potential including different form-factors, the so called Groningen potential. On the other hand as - a more technical point - in Ref. 10] the Thompson equation is solved in full momentum-spin space by the use of Padé approximants, for further details see, e.g., Ref. 12]. For densities above saturation density the self-energies calculated with the Groningen potential are roughly the same as the ones obtained by us, however, in the low density range they are much smaller in magnitude.

\section{3. momentum dependence}

Up to now we have discussed the self-energy of a nucleon in nuclear matter obtained at the Fermi surface $\left(|\mathbf{k}|=k_{F}\right)$, i.e., the density dependence of the self-energy. In the relativistic Brueckner theory the self-energy at the Fermi surface 
and the T-matrix are determined self-consistently, as described above. The dressed nucleon propagators respectively the self-consistent spinor basis, Eq. (9), entering into the calculation of the T-matrix are constructed with the effective mass, Eq. (10). $M^{*}$ itself depends on the density dependent self-energies $\Sigma_{s, 0, v}\left(k_{F}\right)$. Thus only the effective mass acts as a self-consistency or iteration parameter. In a next step the self-energies above and below the Fermi surface can be evaluated from Eqs. (18-20). This has to be considered just as the first iteration step in the determination of self-consistent momentum dependent functions $\Sigma_{s, 0, v}(|\mathbf{k}|)$ and stands in contrast to the non-relativistic Brueckner treatment where self-consistency is required for the full positive single particle energy spectrum. However, these approximations are not based on the same footing. In the relativistic treatment we have an intrinsic momentum dependence due to the Dirac structure of the nucleon which introduces a momentum dependence in the single particle potential $U$ even for constant values of $\Sigma_{s, 0, v}$, see Eq. (27). A further argument often given in connection with the reference spectrum approximation [7, 10] is that the momentum dependence of the self-energies in the relativistic Brueckner approach is found to be rather soft. However, in the present work we demonstrate that this is in general not the case but strongly depends on the special form of the one-boson exchange potential. In Fig. 6 we show the momentum dependence of the nucleon self-energies $\Sigma_{s, 0}$ at saturation density calculated with the $\sigma \omega$-exchange potential. Our results nicely agree with the results of Ref. [7] within $10 \mathrm{MeV}$ which might be due to different technical details already mentioned in the discussion of Fig. 1. We find that $\Sigma_{s}$ decreases in the range from $|\mathbf{k}|=0$ to $k_{F}$ by 14 $\mathrm{MeV}$ and $\Sigma_{0}$ by $24 \mathrm{MeV}$, i.e., below the Fermi surface the self-energies are constant within a few percent.

In Fig. 7 we show the momentum dependence of the self-energies for calculations with the Bonn C potential (pseudovector choice) and the $\sigma \omega$-potential (pseudo-scalar choice), both at $k_{F}=0.265 \mathrm{GeV}$. More strictly this is the real part of the self-energy since for momenta greater than the Fermi momentum the self-energy is complex. The results obtained with the Bonn $\mathrm{C}$ potential show a strong momentum dependence: over the entire momentum range up to $0.8 \mathrm{GeV}$ corresponding to a single particle energy of around $300 \mathrm{MeV}$ the self-energies decrease by nearly $60 \%$ with respect to the central value. The most pronounced change occurs in the region around the Fermi surface while deep inside the Fermi sea and far above the Fermi momentum the momentum dependence nearly saturates. For the $\sigma \omega$-potential the self-energies are rather weakly momentum dependent: over the full interval they decrease by about 20\%. For comparison we also show two results obtained by the Groningen group. In Ref. [10] the self-energies above the Fermi surface are calculated in a similar way as in the present work, see discussion of Fig. 5 . The results obtained by the Groningen group [10] are in general softer k-dependent than those of the present work obtained with the Bonn potentials. The results of Ref. [17] show a momentum dependence as strongly as ours. However, in this case a comparison is not fully appropriate since these calculations include an additional self-consistent treatment of the pion polarisation.

\section{B. equation of state and optical potential}

In Fig. 8 we show the equation of state, i.e., the energy per particle E/A as a function of the nuclear matter density $\varrho$, calculated with Bonn A,B,C. In the relativistic Brueckner theory the energy per particle is defined in analogy to the non-relativistic Hartree-Fock method as the kinetic plus half of the potential energy

$$
\begin{aligned}
& \mathrm{E} / \mathrm{A}=\frac{1}{\varrho} \sum_{\mathbf{k}, \lambda}<\bar{u}_{\lambda}(\mathbf{k})\left|\boldsymbol{\gamma} \cdot \mathbf{k}+M+\frac{1}{2} \Sigma(k)\right| u_{\lambda}(\mathbf{k})>\frac{M^{*}}{E^{*}}-M \\
& =\frac{1}{\varrho} \int_{F} \frac{d^{3} \mathbf{k}}{2 \pi^{3}}\left[\left(\left(1+\Sigma_{v}(|\mathbf{k}|)\right) E^{*}-\Sigma^{0}(|\mathbf{k}|)\right)-\frac{1}{2 E^{*}}\left(\Sigma_{s}(|\mathbf{k}|) M^{*}-\Sigma_{\mu}(|\mathbf{k}|) k^{* \mu}\right)\right]-M
\end{aligned}
$$

with the self-consistent spinors $\mid u_{\lambda}(\mathbf{k})>$ given in Eq. (9). By this way we have determined E/A by integrating the self-energy components of Eqs. (18-20) over the Fermi sphere, see Eq. (29). In Fig. 8 we compare to the results of Ref. [9] where E/A is calculated directly from the single particle potential, Eq. (26), using the expression (28). For moderate densities the results agree with an accuracy better $0.5 \mathrm{MeV}$ and even for $k_{F}=1.9 \mathrm{fm}^{-1}$ the deviation is less than $3 \mathrm{MeV}$. Firstly, this result is an additional test for the accuracy of our numerical treatment, i.e., the projection of the T-matrix onto covariant amplitudes which are subsequently used to determine the self-energy components and the equation of state. Secondly, these results again demonstrate that the equation of state is quite insensitive to the self-energy components themselves. Actually, as can be seen clearly from the definition Eq. (28), the equation of state depends directly on the single-particle potential, i.e., the difference of $\Sigma_{s}$ and $\Sigma_{0}$, Eq. (27), and relatively weak on the effective mass via the kinetic energy part. Nevertheless, the relativistic effects are responsible in contrast to non-relativistic calculations that the minima of the equations of state for the three different Bonn potentials reveal a Coester band which meets the empirically found saturation area of nuclear matter.

In order to demonstrate the influence of the two different choices, i.e., pseudo-vector and pseudo-scalar on the equation of state in Fig. 9 we show the respective equation of state, again for Bonn C. Actually the expectation value 
$\langle\bar{u}|\Sigma| u>$ of the full self-energy operator, Eq. (17), is identical for both choices [26]. However, as can be seen from Fig. 9 the equation of state turns out to be rather sensitive on the different choices. This significant dependence is only due to the self-consistently iterated effective mass which differs in both approaches. This effect deepens the equation of state in the pseudo-scalar choice by around 1 to $2 \mathrm{MeV}$ and shifts the minimum towards higher densities. Thus the saturation point is shifted away from the empirical point towards the results of non-relativistic Brueckner calculations as, e.g., published in Ref. [9]. This observation additionally supports the pseudo-vector choice.

In view of the strong momentum dependence of the self-energy components for the Bonn potentials one might expect that the results depend sensitive on the actual momentum reference value where the selfconsistent effective mass is determined. As a consequence a selfconsistent treatment of the full momentum dependence might appear to be indispensable. Therefore we have also examined the influence of a different choice of the selfconsistent effective mass. In the reference spectrum approximation, see Eq. (10), $M^{*}$ is determined at the Fermi surface which is the conventional treatment in the relativistic Brueckner approach. However, this choice is by no means unique. Thus we also determine $M^{*}$ by its mean value in analogy to Eq. (10)

$$
M^{*}=M+\bar{\Sigma}_{s}\left(M^{*}\right)-M^{*} \bar{\Sigma}_{v}\left(M^{*}\right) .
$$

The self-energies $\Sigma_{s, v}$ are not simply taken at $|\mathbf{k}|=k_{F}$ but replaced by their values averaged over the Fermi sea

$$
\bar{\Sigma}_{s, v}=\frac{\int \Sigma_{s, v}(|\mathbf{k}|) \Theta\left(k_{F}-|\mathbf{k}|\right) M^{*} / E^{*} d^{3} \mathbf{k}}{\int \Theta\left(k_{F}-|\mathbf{k}|\right) M^{*} / E^{*} d^{3} \mathbf{k}} .
$$

These averages are Lorentz scalars. The extension of the reference spectrum approximation, Eq. (30), is especially meaningful with respect to the treatment of anisotropic nuclear matter, as done in Ref. 20]. Here it serves as a test of the influence of the momentum dependence. Due to the strong momentum dependence observed, e.g., in Fig. 7 the mean value $\bar{\Sigma}_{s}$ differs considerably from $\Sigma_{s}\left(k_{F}\right)$, i.e., by about $57 \mathrm{MeV}$ at saturation density (with Bonn C). Respectively for the different effective masses we obtain a mean value $M^{*}=528 \mathrm{MeV}$ which is smaller than the selfconsistent mass $M^{*}\left(k_{F}\right)=566 \mathrm{MeV}$. But if we use the alternatively defined effective mass, Eq. (30), as iteration parameter we find for the final iterated mean value $M^{*}=547 \mathrm{MeV}$. Thus the mean value is increased, i.e., it is shifted towards the previous self-consistent result or, in other words, the selfconsistent value is rather stable. The comparison of the self-energy components $\Sigma_{s, 0}\left(k_{F}\right)$ in both iterations, see Fig. 2, shows just slightly deviations, e.g., about 10 $\mathrm{MeV}$ at saturation density. And even more, the equation of state, shown in Fig. 9, remains nearly unchanged. These results demonstrate that the strong momentum dependence has only minor influence on the density dependence of the self-energy components and that the equation of state remains unaffected.

Another quantity which is more sensitive on the momentum dependence of $\Sigma_{s, 0, v}$ is the Schroedinger equivalent optical potential which a nucleon feels inside the nuclear medium. The optical potential is given by

$$
U_{\mathrm{opt}}\left(|\mathbf{k}|, k^{0}\right)=\Sigma_{s}(|\mathbf{k}|)-\frac{1}{M} k^{\mu} \Sigma_{\mu}(|\mathbf{k}|)+\frac{\Sigma_{s}^{2}(|\mathbf{k}|)-\Sigma_{\mu}^{2}(|\mathbf{k}|)}{2 M} .
$$

It contains counteracting linear and quadratic terms in the self-energy components. For nucleons with $|\mathbf{k}|>k_{F}$ the momentum space is not completely blocked for scattering processes and thus above the Fermi surface the self-energies and the optical potential become complex. The single-particle energy $k^{0}(|\mathbf{k}|)=-\Sigma^{0}+E^{*}(|\mathbf{k}|)$ is a monotonic function of $|\mathbf{k}|$ which allows by inversion to determine $U_{\text {opt }}\left(k^{0}\right)$ as function of the single-particle energy $k^{0}$. We want to stress that neglecting the momentum dependence of the fields $\Sigma_{s,(0)}$ and neglecting completely $\Sigma_{v}$ the optical potential $U_{\text {opt }}$ would simply be a linear function of $k^{0}$. However, this is not the case here. Real and imaginary part of the optical potential calculated for Bonn C (pseudo-vector choice) are shown in Fig. 10. The real part agrees well with the Groningen results of Ref. 11] while the imaginary part of the present calculations is about twice as large. Further we compare to the empirical optical potential Hama I, table 2 of Ref. [28]. For the full range of single-particle energies up to $700 \mathrm{MeV}$, shown in Fig. 10, our results deviate from the empirical $\operatorname{Re} U_{\text {opt }}$ by less than $10 \mathrm{MeV}$. Up to the pion production threshold at around $300 \mathrm{MeV}$ we observe a remarkable agreement of the imaginary part of $U_{\text {opt }}$ with the empirical data. For single-particle energies above this region the results appear to be no more fully reliable since then meson-nucleon resonances, e.g. the $\Delta(1232)$ resonance, should be taken into account.

\section{SUMMARY}

In this work in particular the self-energy of a nucleon inside the nuclear medium has been studied in the framework of the relativistic Brueckner approach. Therefore the dependence of the Lorentz scalar and vector self-energy components 
on the nuclear density and the nucleon momentum have been examined. As bare nucleon-nucleon interaction various one-boson exchange potentials have been employed, i.e., the Bonn A, B, C potentials and for comparison the $\sigma \omega-$ exchange potential. For the latter we are able to nicely reproduce the density dependence of the self-energies of Ref. 17. The self-energy components themselves deviate for the three different Bonn potentials over a wide density range only by a few $\mathrm{MeV}$ and thus we restricted our discussion mainly to the Bonn $\mathrm{C}$ potential. From the comparison with our results we find that the potential-fit method of Ref. [9] underpredicts the magnitude of the self-energies by about 50 up to more than $150 \mathrm{MeV}$.

Further we analyzed the influence of different possible covariant representations of the T-matrix. The positive energy on-shell T-matrix is projected on five invariant amplitudes and by the restriction to positive energy states an ambiguity arises which is discussed in terms of the pseudo-scalar and the pseudo-vector representation. Although the T-amplitudes of both choices are equal on the one-body level, i.e., concerning the respective self-energy components a significant influence of these representations is observed. This is due to the fact that in the pseudo-scalar case direct and exchange contributions to the self-energies are equal while in the pseudo-vector choice the exchange part is suppressed. For the Bonn potentials this suppression lies between $40-80 \mathrm{MeV}$ and is most prominant at low densities. This difference between both choices has to be interpreted as an inherent measure for an uncertainty of the approach due to the restriction on positive energy spinors.

Commonly the momentum dependence of the self-energy components is supposed to be weak. This assumption is also reflected in the self-consistency treatment in the relativistic Brueckner model which is managed by just one density dependent parameter, i.e., the self-consistent effective mass. Therefore we have carefully examined this momentum dependence which, however, can be done within the Bruckner scheme just in a first approximation. As in Ref. [7] we find that taking the $\sigma \omega$-exchange potential the self-energies are found to be constant below the Fermi surface within a few percent. However, this result does not hold for the more realistic Bonn potentials. Here we observe a strong momentum dependence which may be due to the strong pion exchange. The self-energies decrease from their central value, i.e., at $k=0$ to the Fermi surface by about one fourth and further to $k=0.8 \mathrm{GeV}$ by more than one half. This striking momentum dependence makes the treatment of the self-consistency in the model somewhat questionable. But a test with a different defined self-consistent effective mass which is determined by a mean value over the Fermi sea and not taken at $k_{F}$ as usually done shows that the self-energy components are rather stable at not too high densities. Thus the self-consistency treatment in the model still appears to be justified up to two or three times saturation density.

In this context the question raises which observables are sensitive to the self-energy components and their momentum dependence. It is remarkable that the full self-energy and, respectively, the single particle potential show (absolutely) a much weaker momentum dependence. As it is well known from mean field theory the large scalar and vector selfenergy components contribute to these quantities with opposite signs and thus cancel each other to hight extent. The resulting observables, e.g., the single particle potential are smaller by nearly one order of magnitude. Here we found that this holds not only at $k_{F}$ but also over the full momentum range considered. Thus the momentum dependence cancels to a large extent. Also the equation of state depends mainly on the difference of the scalar and the vector self-energy and thus the absolute values of the single component are of minor importance. This can be seen most clearly by the use of just density dependent self-energy components determined with the potential-fit method of Brockmann and Machleidt [9] which deviate from our results (at $k_{F}$ ) by about $75 \mathrm{MeV}$. The resulting binding energy, however, only deviates by about $1 \mathrm{MeV}$. Similarly the self-energy components obtained in the pseudo-scalar and the pseudo-vector choice differ considerable but the equations of state differ by less than one to two MeV. On the other hand, small variations of the self-energy due to the different three Bonn potentials produce a significant change in the equations of state. Summarizing, the equation of state is not sensitive to the self-energy components themselves but to their difference. A bit more sensitive is another quantity, i.e., the optical potential. It depends not only on the cancellation of the scalar and vector self-energy but also contains quadratic terms. We find a good agreement of our results with the experimental data of Ref. [28] for, both, the real and the imaginary part up to a single particle energy of several hundred $\mathrm{MeV}$.

As a resumé, the self-energy components in the relativistic Brueckner model are still affected with some uncertainty. For realistic one-boson exchange potentials we find them to be strongly momentum dependent. However, for this attribute we have to be aware of the limits of the model. One major success of the relativistic approach was that the equation of state comes close to the empirical saturation point. The equation of state is rather insensitive to the self-energy components themselves. Similarly, the nucleon optical potential is only little more sensitive. However, a precise knowledge of the size of each self-energy component is necessary, e.g., in the much more complicate situation of heavy ion collisions. 


\section{ACKNOWLEDGMENTS}

The authors acknowledge stimulating discussions with F. de Jong and H. Müther.

[1] J. D. Walecka, Ann. Phys. (N. Y.) 83, 497 (1974);

B. D. Serot, J. D. Walecka, Advances in Nuclear Physics, 16, 1, eds. J. W. Negele, E. Vogt, (Plenum, N.Y., 1986)

[2] G. E. Brown, A. D. Jackson, The Nucleon Nucleon Interaction (North Holland, Amsterdam, 1976).

[3] R. Machleidt, Advances in Nuclear Physics, 19, 189, eds. J. W. Negele, E. Vogt, (Plenum, N.Y., 1989).

[4] M. R. Anastasio, L. S. Celenza, C. M. Shakin, Phys. Rev. C23, 2258 (1981); Phys. Rev. C23, 2273 (1981).

[5] M. R. Anastasio, L. S. Celenza, W. S. Pong, C. M. Shakin, Phys. Rep. 100, 327 (1983).

[6] C. J. Horowitz, B. D. Serot, Phys. Lett. B137, 287 (1984).

[7] C. J. Horowitz, B. D. Serot, Nucl. Phys. A464, 613 (1987).

[8] R. Brockmann, R. Machleidt, Phys. Lett. B149, 283 (1984).

[9] R. Brockmann, R. Machleidt, Phys. Rev. C42, 1965 (1990).

[10] B. t. Haar, R. Malfliet, Phys. Rep. 149, 207 (1987).

[11] B. t. Haar, R. Malfliet, Phys. Rev. C36, 1611 (1987).

[12] R. Malfliet, Progress in Particle and Nuclear Physics, 21, 207, ed. A.Faessler, (Oxford, 1988);

W. Botermans, R. Malfliet, Phys. Rep. 198, 115 (1990);

F. d. Jong, R. Malfliet, Phys. Rev. C44, 998 (1991).

[13] M. I. Haftel, F. Tabakin, Nucl. Phys. A158, 1 (1970).

[14] P. Poschenrieder, M. K. Weigel, Phys. Rev. C38, 471 (1988).

[15] C. Nuppenau, Y. J. Lee, A. D. MacKellar, Nucl. Phys. A504, 839 (1989).

[16] J. A. Tjon, S. J. Wallace, Phys. Rev. C32, 267 (1985); Phys. Rev. C32, 1667 (1985).

[17] F. d. Jong, B. t. Haar, R. Malfliet, Phys. Lett. B220, 485 (1989).

[18] C. Fuchs, H. Lenske, H. H. Wolter, Phys. Rev. C52, 3043 (1995).

[19] C. Fuchs, E. Lehmann, L. Sehn, F. Scholz, T. Kubo, J. Zipprich, A. Faessler, Nucl. Phys. A603, 471 (1996).

[20] L. Sehn, H. H. Wolter, Nucl. Phys. A601, 473 (1996);

C. Fuchs, L. Sehn, H. H. Wolter, Nucl. Phys. A601, 505 (1996);

H. Elsenhans, L. Sehn, A. Faessler, H. Müther, N. Ohtzuka, H. H. Wolter, Nucl. Phys. A536, 750 (1992).

[21] C. Fuchs, T. Gaitanos, H. H. Wolter, Phys. Lett. B381, 23 (1996).

[22] K. Erkelenz, Phys. Rep. C13, 191 (1974).

[23] J. D. Bjorken, S. D. Drell, Relativistic Quantum Mechanics (McGraw-Hill, N.Y., 1964);

J. D. Bjorken, S. D. Drell, Relativistic Quantum Fields (McGraw-Hill, N.Y., 1965).

[24] M. Trefz, A. Faessler, W. H. Dickhoff, Nucl. Phys. A443, 499 (1985).

[25] M. Rose, Elementary Theory of Angular Momentum (Wiley, N.Y., 1957).

[26] F. d. Jong, Ph.D. thesis, Groningen, The Netherlands (1992) (unpublished).

[27] R. Machleidt, in: Computational Nuclear Physics 2 - Nuclear Reactions, K. Langanke, J. A. Maruhn, and S. E. Koonin, eds. (Springer, New York, 1993), Chapter 1, p. 1.

[28] S. Hama, B. C. Clark, E. D. Cooper, H. S. Sherif, R. L. Mercer, Phys. Rev. C41, 2737 (1990). 


\section{FIGURE CAPTIONS}

Fig. 1: Self-consistent effective mass $M^{*}$ in units of the bare mass $(M=939 \mathrm{MeV})$ as a function of the Fermi momentum $k_{F}$. The relativistic Brueckner results obtained with the $\sigma \omega$-exchange potential (full line) are compared to those of Ref. If] (diamonds). The effective mass for the Bonn $\mathrm{C}$ potential (dashed line) differs significantly from that of the $\sigma \omega$-model.

Fig. 2: Self-energy components for the pseudo-vector choice, calculated with various Bonn potentials. The dotted, dashed and full lines represent the Bonn A,B and C potential. The scalar self-energy $\Sigma_{s}$ (lower half-plane) and the time- and space-like vector self-energies $-\Sigma_{0},-k_{F} \Sigma_{v}$ (upper half-plane) are shown as functions of $k_{F}$. For the three Bonn potentials the small space-like vector self-energies $-k_{F} \Sigma_{v}$ are equal within line width. The remaining curves (dashed dotted) involve a different definition of the self-consistent effective mass which is given in the text later on.

Fig. 3: Self-energy components $\Sigma_{s},-\Sigma_{0}$ calculated with the pseudo-scalar choice and the Bonn C potential. The curves represent the total self-energy components (full lines), their direct (dir.) and their exchange (exc.) parts which are practically equal (dashed dotted lines).

Fig. 4: Self-energy components $\Sigma_{s},-\Sigma_{0}$ calculated with the pseudo-vector choice and the Bonn C potential. The curves represent the total self-energy components (full lines), their direct (dir.) parts (dashed lines) and their exchange (exc.) parts (dotted lines).

Fig. 5: Self-energy components $\Sigma_{s},-\Sigma_{0}$ calculated with the pseudo-vector choice and the Bonn C potential (full lines). They are compared with the results of Ref. [9] (dashed lines) and of Ref. [10] (dotted lines).

Fig. 6: Self-energy components $\Sigma_{s},-\Sigma_{0}$ at saturation density $\left(k_{F}=0.28 \mathrm{GeV}\right)$ as function of the nucleon momentum $|\mathbf{k}|<k_{F}$. The self-energies are calculated with the $\sigma \omega$-exchange potential (pseudo-scalar choice) and compared with values (symbols) taken from Ref. [7].

Fig. 7: Self-energy components $\Sigma_{s},-\Sigma_{0}$ as function of the momentum $|\mathbf{k}|$ of the nucleon for $k_{F}=0.265$ GeV. The self-energies are calculated with the Bonn $\mathrm{C}$ (pseudo-vector choice, solid line) and the $\sigma \omega$-exchange potential (pseudo-scalar choice, dotted line) and compared with results of Ref. [10] (dashed line) and of Ref. 17] $\left(k_{F}=0.27\right.$ $\mathrm{GeV}$, dashed dotted line).

Fig. 8: Equation of state, i.e., energy per particle $E / A$ as a function of the nuclear matter density $\varrho$, for the three Bonn potentials A,B and $\mathrm{C}$ (from below to top: dashed line, dotted line and solid line). The symbols represent the respective results of Ref. [9].

Fig. 9: Equation of state for the Bonn $\mathrm{C}$ potential calculated in the pseudo-vector (solid line) and the pseudo-scalar (dashed line) choice. The remaining curve (dotted) involves a different definition of the self-consistent effective mass given in the text.

Fig. 10: Real and imaginary part of the Schroedinger equivalent optical potential $U_{\text {opt }}$ as function of the single-paricle energy $k^{0}-M$. The results for the Bonn $\mathrm{C}$ potential (real part: solid line, imaginary part: dotted line) are compared to the results of Ref. [10] (real part: dashed line, imaginary part: dashed dotted line) and to the empirical optical potential taken from Ref. 28] (real part: diamonds, imaginary part: circles). 


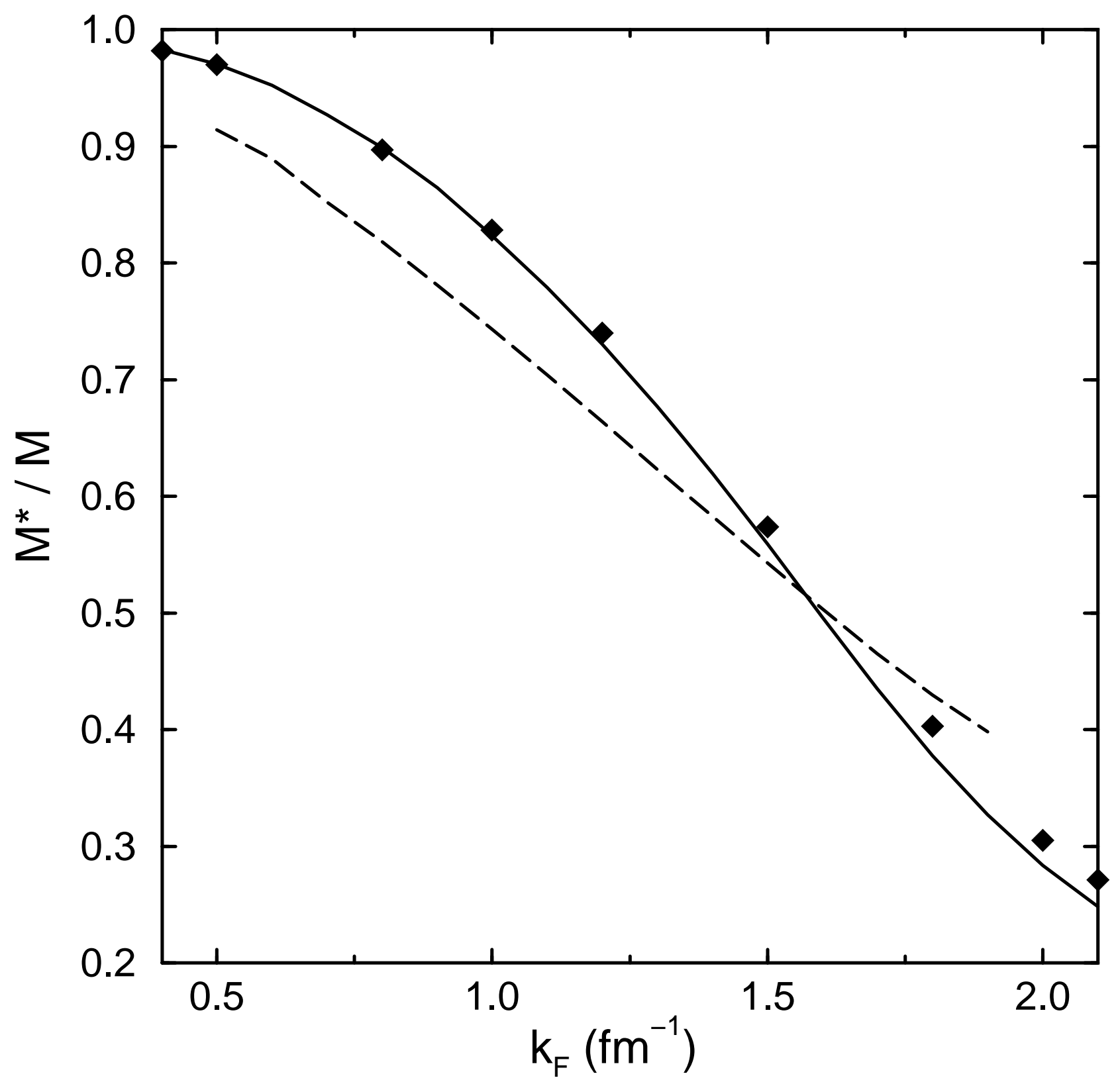

FIG. 1. 


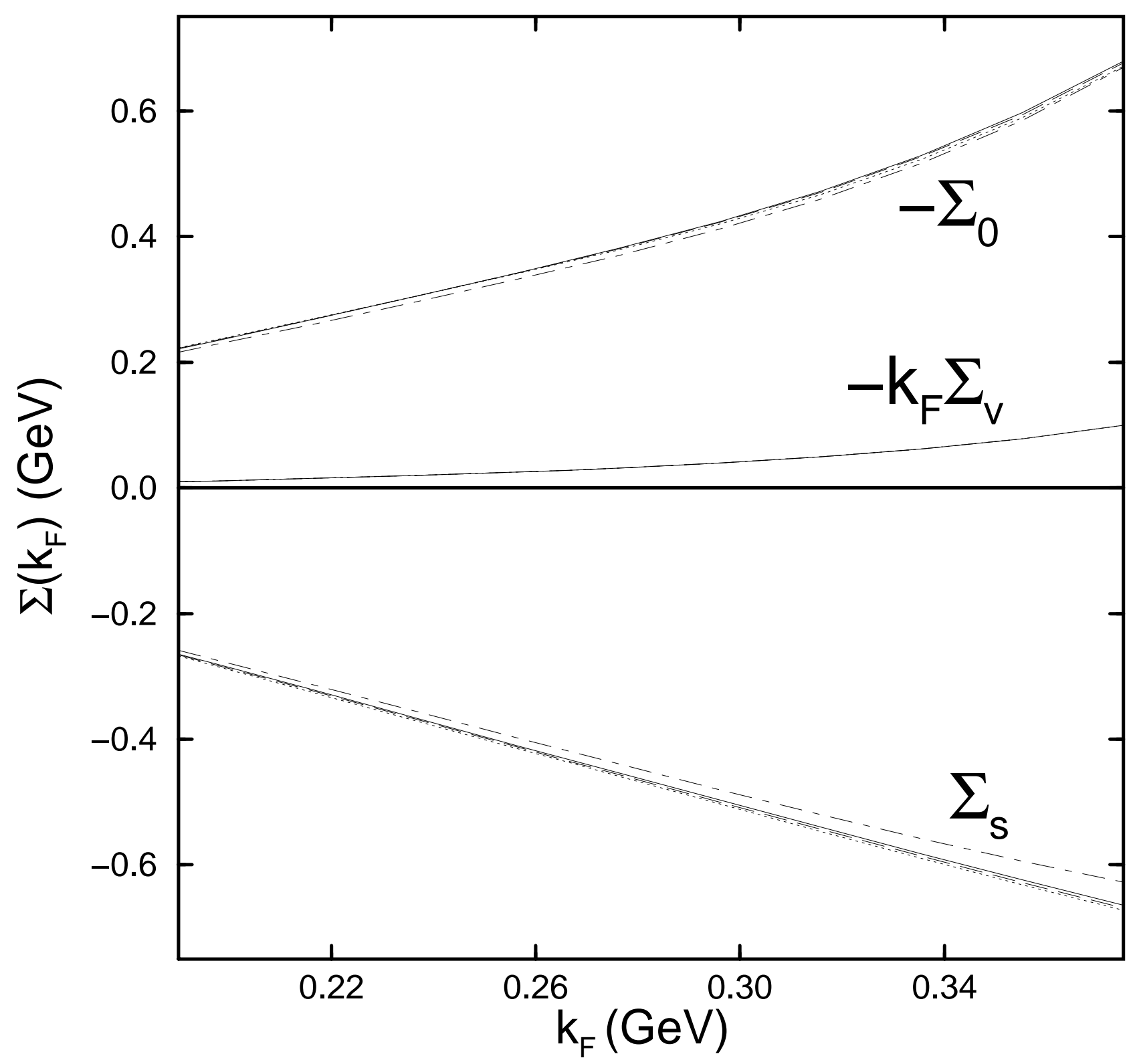

FIG. 2. 


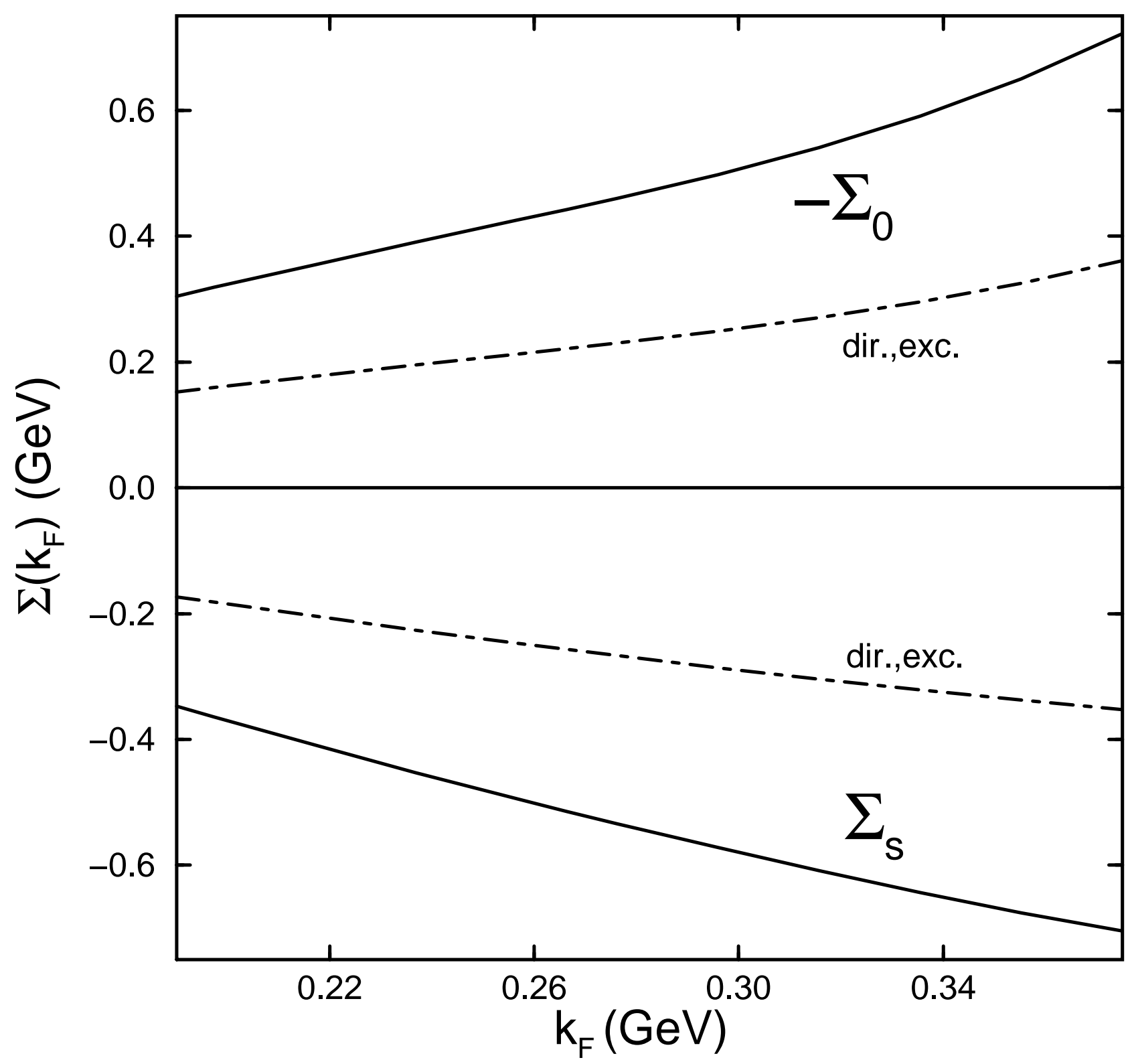

FIG. 3 


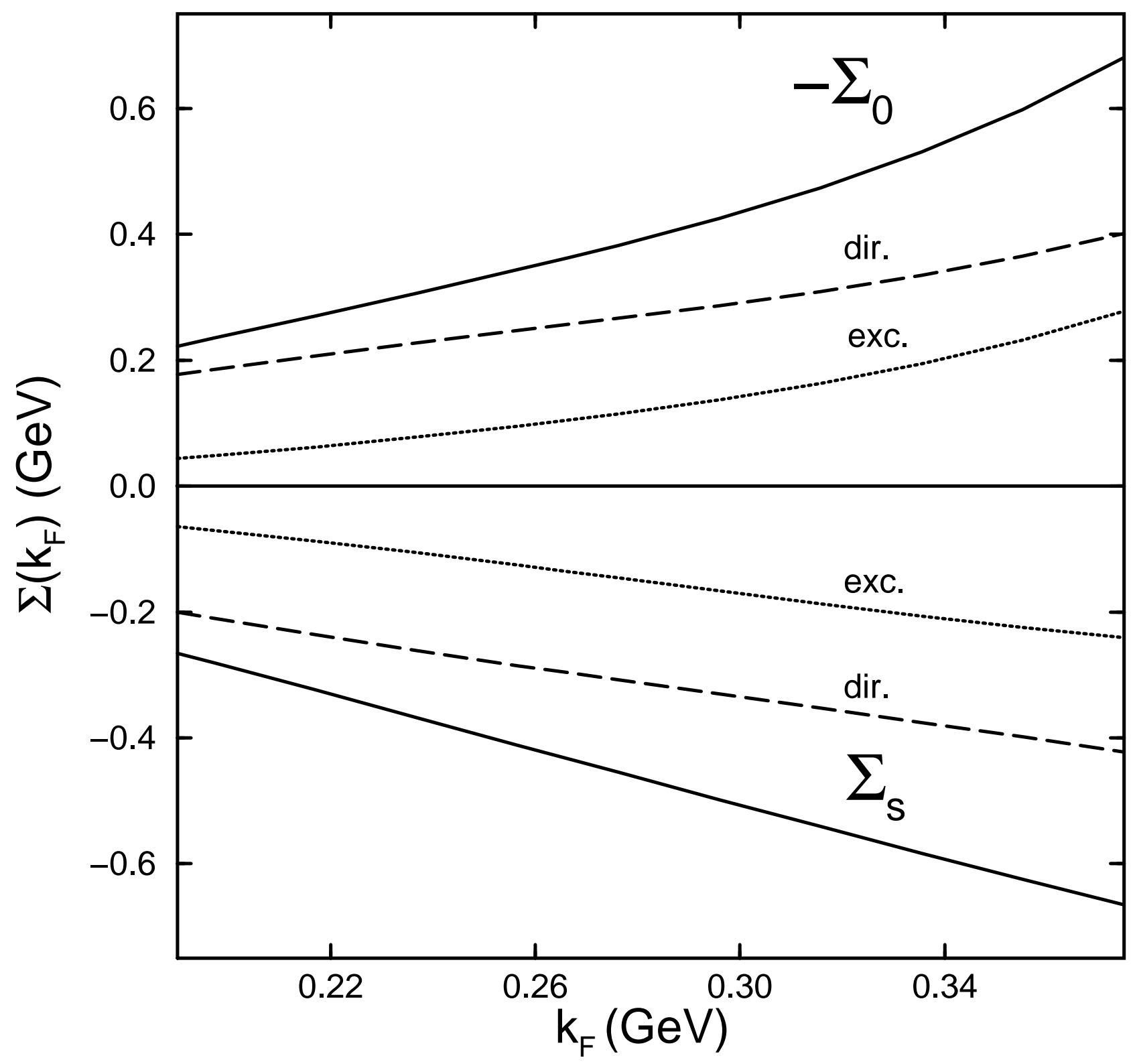

FIG. 4 


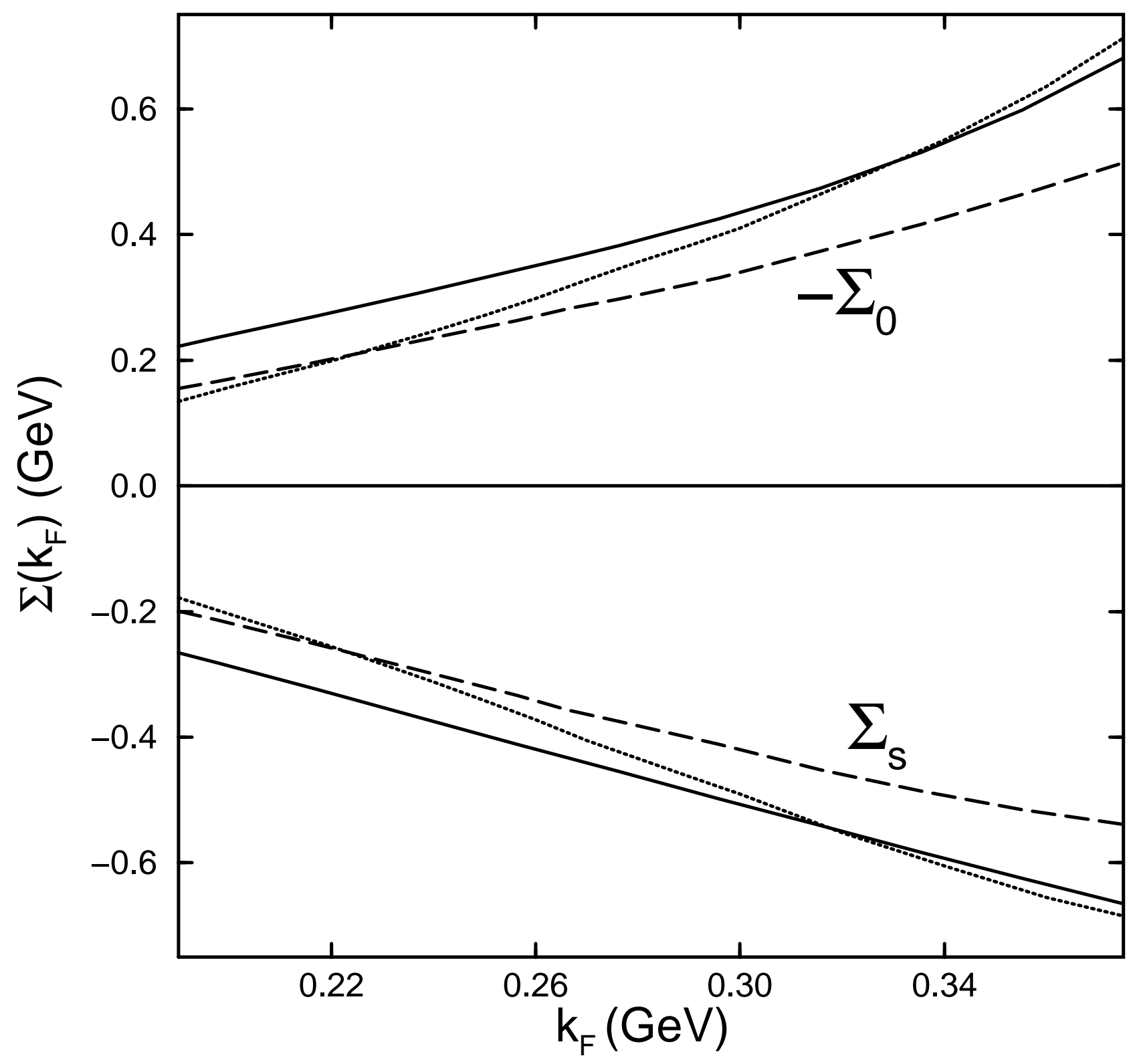

FIG. 5 . 


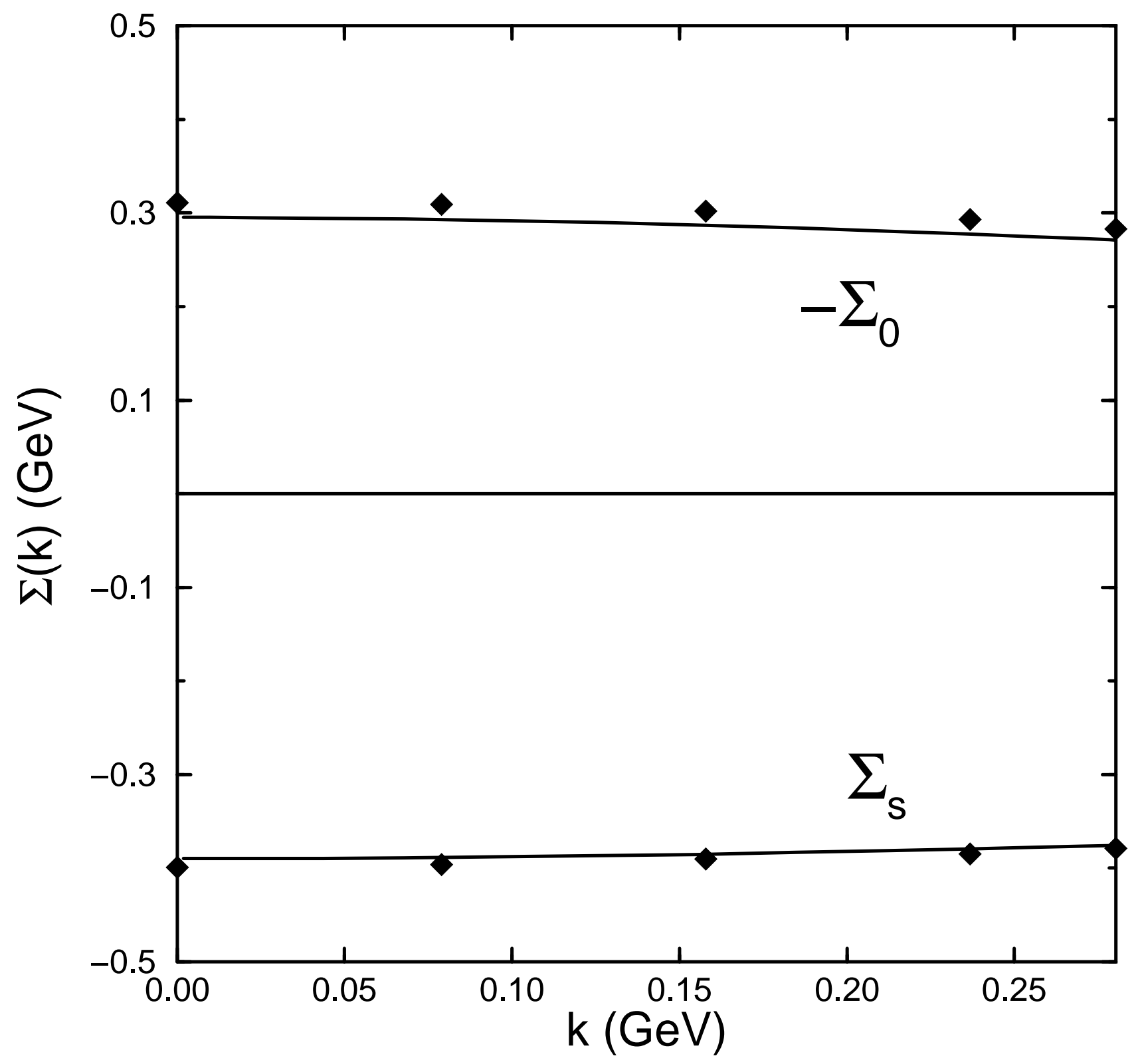

FIG. 6. 


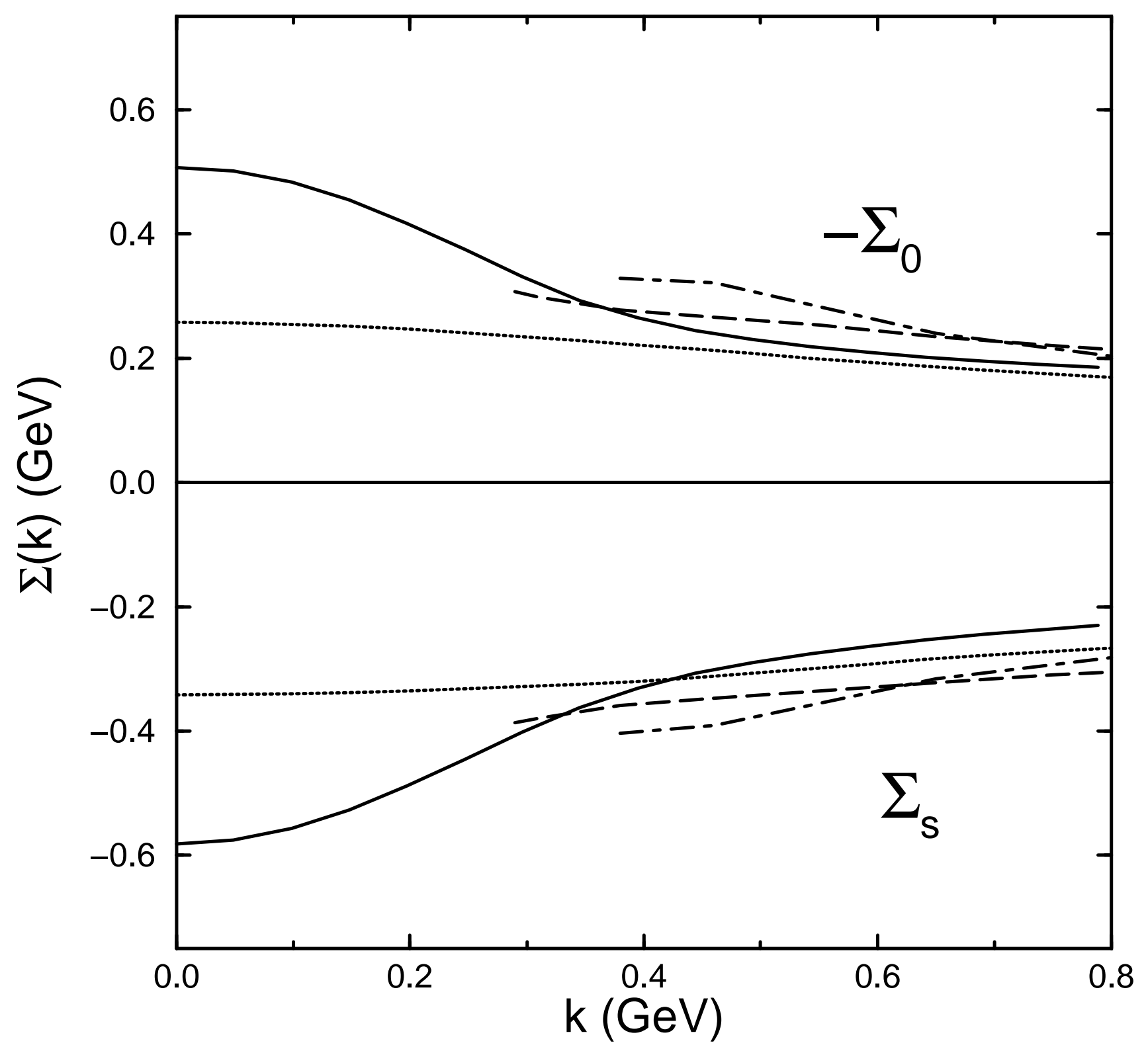

FIG. 7. 


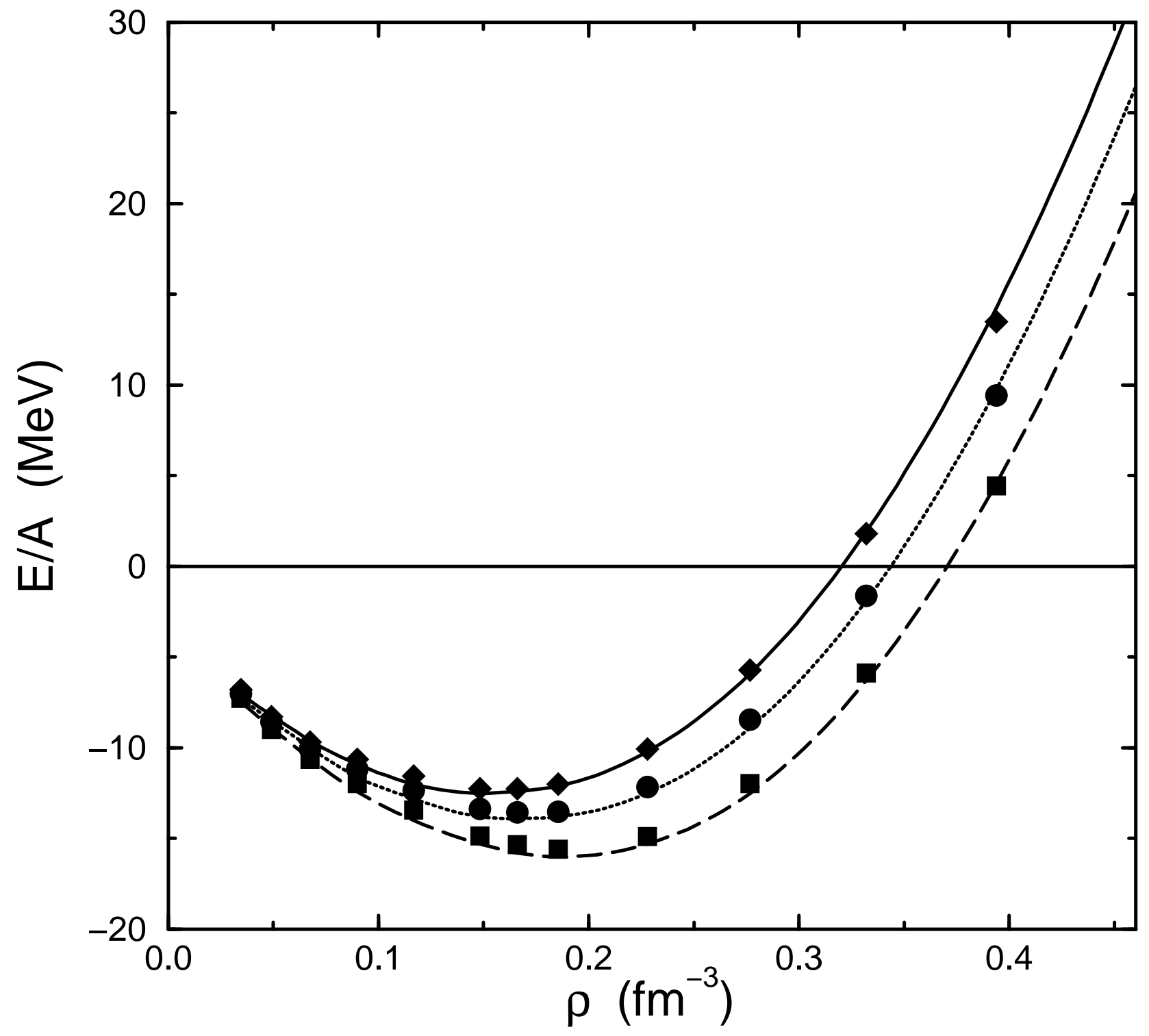

FIG. 8. 


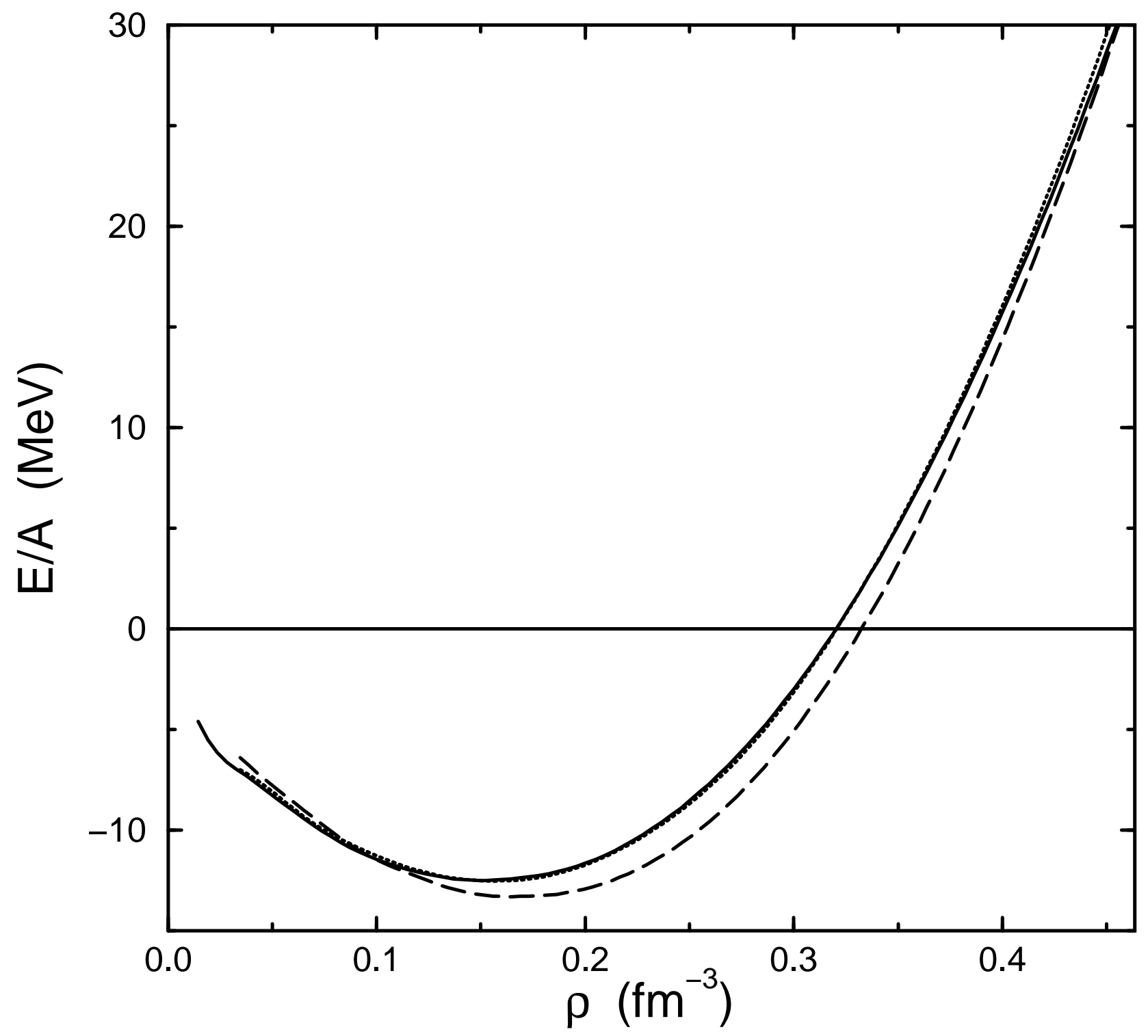

FIG. 9. 


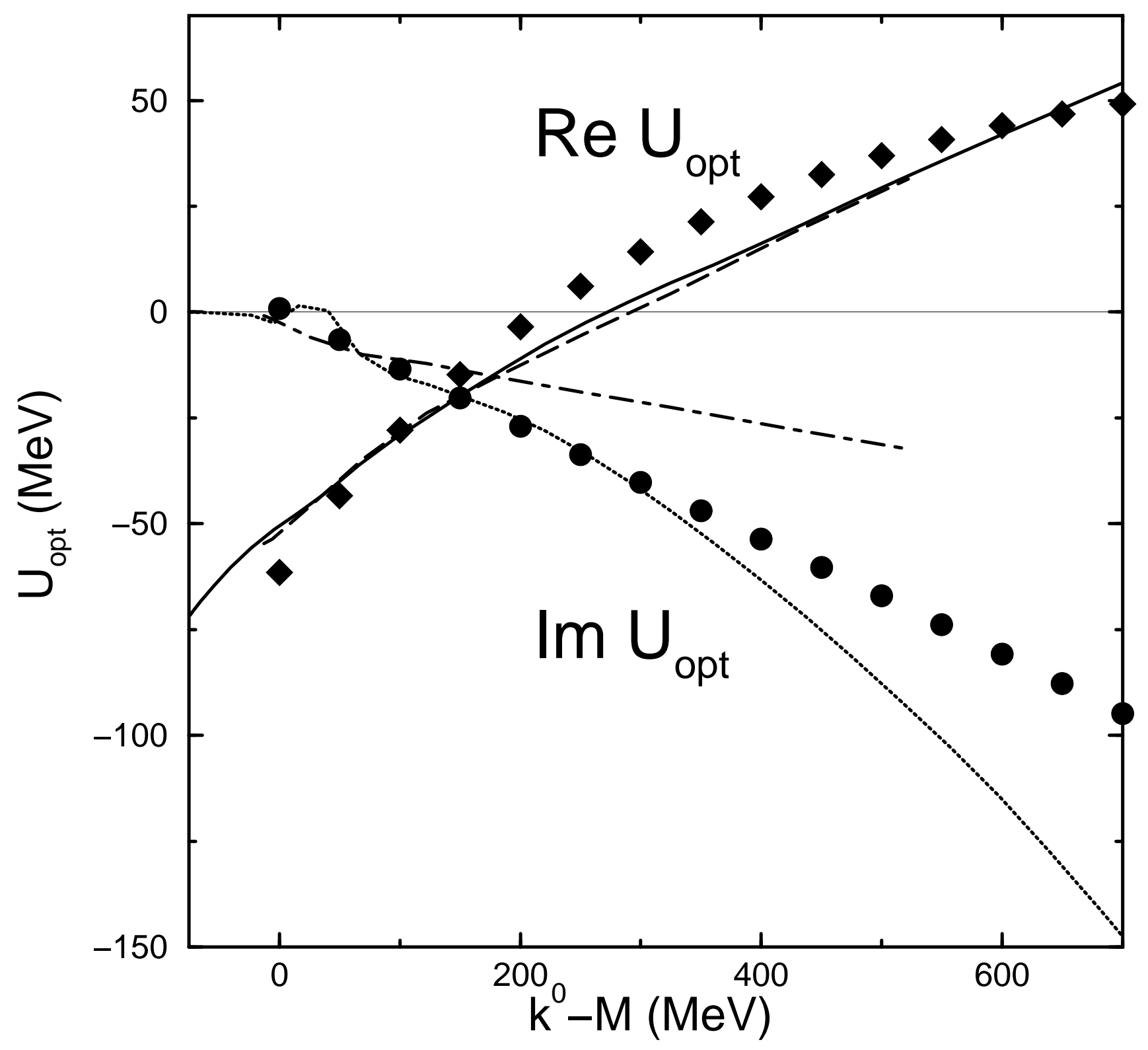

FIG. 10. 